\title{
The Neural Architecture of General Knowledge
}

\author{
ERHAN GENÇ ${ }^{1 *}{ }^{*}$ CHRISTOPH FRAENZ ${ }^{1}$ DD, CAROLINE SCHLÜTER ${ }^{1}$, PATRICK FRIEDRICH $^{1}$, \\ MANUEL C. VOELKLE ${ }^{2}$, RÜDIGER HOSSIEP ${ }^{3}$ and ONUR GÜNTÜRKÜN ${ }^{1,4}$ \\ ${ }^{1}$ Department of Psychology, Biopsychology, Ruhr University Bochum, Bochum, Germany \\ ${ }^{2}$ Department of Psychology, Psychological Research Methods, Humboldt University Berlin, Berlin, Germany \\ ${ }^{3}$ Department of Psychology, Team Test Development, Ruhr University Bochum, Bochum, Germany \\ ${ }^{4}$ Stellenbosch Institute for Advanced Study (STIAS), Wallenberg Research Centre at Stellenbosch University, Stellenbosch, South Africa
}

\begin{abstract}
Cognitive performance varies widely between individuals and is highly influenced by structural and functional properties of the brain. In the past, neuroscientific research was principally concerned with fluid intelligence, while neglecting its equally important counterpart crystallized intelligence. Crystallized intelligence is defined as the depth and breadth of knowledge and skills that are valued by one's culture. The accumulation of crystallized intelligence is guided by information storage capacities and is likely to be reflected in an individual's level of general knowledge. In spite of the significant role general knowledge plays for everyday life, its neural foundation largely remains unknown. In a large sample of 324 healthy individuals, we used standard magnetic resonance imaging along with functional magnetic resonance imaging and diffusion tensor imaging to examine different estimates of brain volume and brain network connectivity and assessed their predictive power with regard to both general knowledge and fluid intelligence. Our results demonstrate that an individual's level of general knowledge is associated with structural brain network connectivity beyond any confounding effects exerted by age or sex. Moreover, we found fluid intelligence to be best predicted by cortex volume in male subjects and functional network connectivity in female subjects. Combined, these findings potentially indicate different neural architectures for information storage and information processing. (C) 2019 European Association of Personality Psychology
\end{abstract}

Key words: general knowledge; intelligence; interindividual differences; DTI; brain network connectivity

\section{INTRODUCTION}

From the day we are born, we acquire knowledge about the world around us. Across our lifespan, we gather a whole treasure of information and master an impressive set of skills by asking, reading, observing, or just trying. The knowledge and abilities obtained in this way are traditionally subsumed under the concept of crystallized intelligence (Cattell, 1963). Among the variety of cognitive abilities, crystallized intelligence takes a special place because it includes both dynamic procedural knowledge and static declarative knowledge (Flanagan, 2013). Procedural knowledge, on the one hand, refers to the canon of learned procedures that allow us to utilize previously acquired knowledge in order to fulfil different tasks. Declarative knowledge, on the other hand, is stored in long-term memory and tends to be verbal in nature (Flanagan, 2013). It comprises different types of static

*Correspondence to: Erhan Genç, Abteilung Biopsychologie, Institut für Kognitive Neurowissenschaft, Fakultät für Psychologie, Ruhr-Universität Bochum, Universitätsstraße 150, 44801 Bochum, Germany.

E-mail: erhan.genc@rub.de

$\dagger$ E. G. and C. F. contributed equally to this manuscript and share the first author position.

This article earned Open Materials badge through Open Practices Disclosure from the Center for Open Science: https://osf.io/tvyxz/wiki. The materials are permanently and openly accessible at https://osf.io/zmw5y/. Author's disclosure form may also be found at the Supporting Information in the online version. information like rules, relationships, abstract concepts, or, most importantly, general knowledge. For example, knowing the street address of the best pizza place in town would be considered declarative knowledge, whereas the act of driving there for lunch would draw on procedural knowledge.

The Gf-Gc theory, conceived by Cattell (1963), is one of the first fundamental theories about the structure of human cognitive abilities. According to this theory, crystallized intelligence constitutes one of the two major factors representing the overall cognitive capacity of an individual. The overall cognitive ability is also referred to as general intelligence or simply $g$. The counterpart to crystallized intelligence, called fluid intelligence, represents all mental operations that come into play when one cannot draw on previously acquired knowledge but has to solve an unfamiliar problem. Over the past decades, the dichotomic concept of cognitive ability being constituted by crystallized and fluid intelligence has been refined by means of factor analytical research. One of the more recent theories, the well-established Cattell-Horn-Carroll (CHC) model (Flanagan, 2013; McGrew, 2005; W. Schneider \& McGrew, 2012; W. J. Schneider \& Newman, 2015), suggests that the structure of cognitive ability can be represented by three hierarchically organized levels or strata. In this model, crystallized and fluid intelligence are referred to as Gc and Gf. Both constructs are placed in stratum II along with several other cognitive abilities. As in Gf-Gc theory, these broad abilities from stratum II 


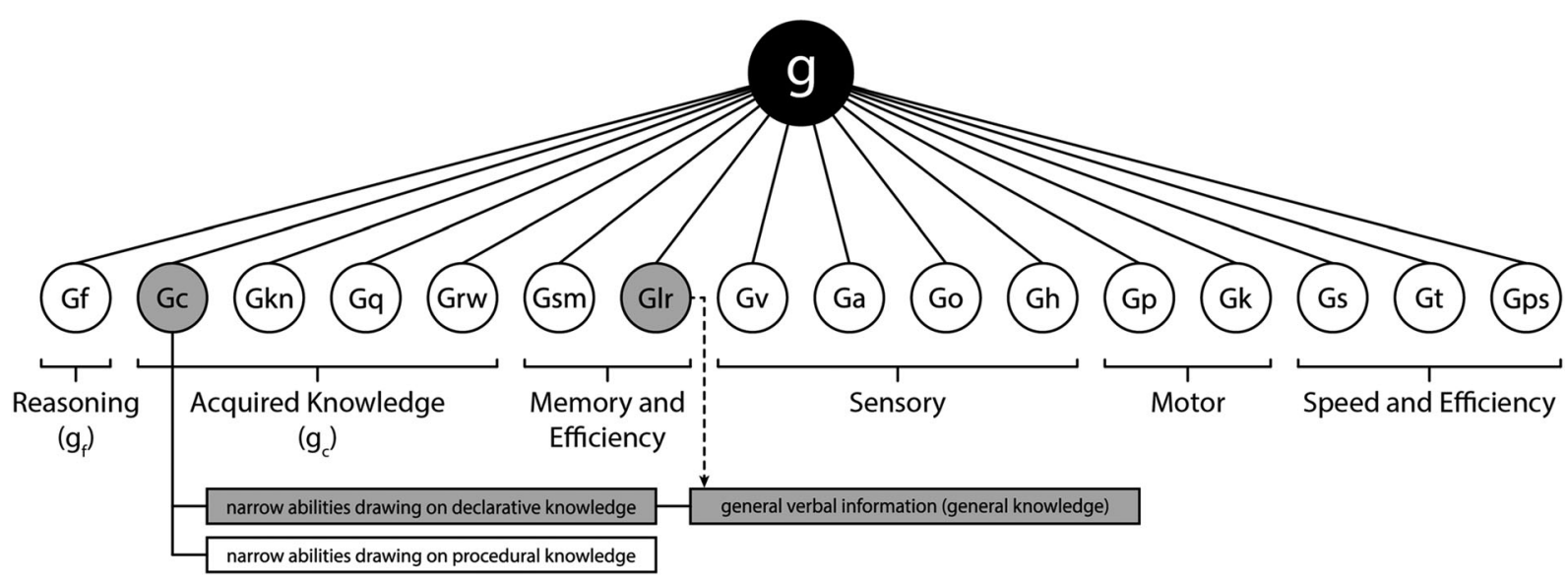

Figure 1. Current Cattell-Horn-Carroll theory of cognitive abilities. The big black circle at the apex of this figure depicts general intelligence from the third stratum. It represents the most abstract level of cognitive ability and subsumes 16 broad abilities from stratum II that are represented by smaller white circles with abbreviations. Each ability from stratum II can be placed into one of six conceptual groupings. Two of these groups, reasoning and acquired knowledge, resemble general fluid $\left(g_{f}\right)$ and general crystallized $\left(g_{c}\right)$ intelligence from Cattell's original Gf-Gc theory (Cattell, 1963). Stratum I includes more than 80 narrow abilities that are largely omitted from this figure because of space limitations. The three boxes at the bottom represent the segmentation of Gc into declarative and procedural knowledge as well as general knowledge. The grey circles and boxes indicate the study's focus on general knowledge and hence on abilities Gc and Glr (see the Discussion section). The dashed arrow represents the involvement of the Glr ability in accumulating general knowledge. Gf, fluid intelligence; Gc, crystallized intelligence; Gkn, domain-specific knowledge; Gq, quantitative knowledge; Grw, reading/writing ability; Gsm, short-term memory; Glr, long-term storage and retrieval; Gv, visual processing; Ga, auditory processing; Go, olfactory abilities; Gh, tactile abilities; Gp, psychomotor abilities; Gk, kinaesthetic abilities; Gs, processing speed; Gt, decision speed/reaction time; Gps, psychomotor speed. The figure was adapted from Flanagan (2013).

load on a common factor, general intelligence or $g$, that is placed at the apex of the model in stratum III (Figure 1). All cognitive abilities from stratum II, including crystallized and fluid intelligence, are treated as higher order factors that are manifested in more narrow abilities that can be quantified by suitable test inventories (W. Schneider \& McGrew, 2012). For fluid intelligence, abilities in deductive and inductive reasoning, which are usually assessed by matrix reasoning tests, are considered to be the most essential indicators of the construct. In the case of crystallized intelligence, underlying narrow abilities are centred around culturally relevant skills and information. Hence, it is a common practice to quantify crystallized intelligence by means of verbal ability tasks such as vocabulary or verbal analogy tests. Several studies also include numerical reasoning as an indicator of crystallized intelligence (Colom et al., 2009; Roman et al., 2014). However, the most important narrow ability within crystallized intelligence is arguably that of general verbal information. It is defined as 'the breadth and depth of knowledge that one's culture deems essential, practical, or otherwise worthwhile for everyone to know' (W. Schneider \& McGrew, 2012). General verbal information refers to acquired knowledge across many domains and can therefore be distinguished from achievement tests or domain-specific tests of more specialized knowledge. Given these definitions, it is feasible to use general verbal information synonymously with a term that is more common in everyday language, namely, general knowledge. Unfortunately, general knowledge has been widely neglected by scientific research in the past, despite being of outmost interest for the general public (Ackerman, 1996; W. Schneider \& McGrew, 2012).

Psychologists are able to estimate the amount of general knowledge held by an individual quite precisely (Ackerman, 1996; Ackerman, Bowen, Beier, \& Kanfer, 2001) and discovered that it is associated with many aspects of everyday life such as occupational success (Ackerman, 1996), physical well-being (Kenkel, 1991) and partner choice (Regan \& Joshi, 2003). In addition to that, mankind's continuous gain in general knowledge of the world is supposed to be the driving force behind its fast-paced cultural evolution (Workman \& Reader, 2014). In fact, we are the intellectual heirs of all of the savants and geniuses throughout history (W. Schneider \& McGrew, 2012). The solutions and ideas they conceived, using their outstanding creative capacity and fluid intelligence, are passed down as general knowledge and add to the crystallized intelligence of future generations (W. Schneider \& McGrew, 2012). As with fluid intelligence, individuals differ with regard to their abilities in the acquisition, storage, and retrieval of general knowledge. This variance is likely to be brought about by interindividual differences in certain brain properties. Despite its importance for our everyday life, the neural foundation of general knowledge largely remains unknown.

Over the last decades, neuroscientific research concerned with the cognitive abilities of the human brain was mainly focused on perceptual performance and fluid intelligence as its objects of investigation (Deary, Penke, \& Johnson, 2010; Kanai \& Rees, 2011). Consequently, fluid intelligence has been linked to a wide variety of neural properties such as brain volume (McDaniel, 2005; Pietschnig, Penke, Wicherts, Zeiler, \& Voracek, 2015), cortical thickness (Karama et al., 2011; Narr et al., 2007), white matter integrity (Penke et al., 2012; Ritchie et al., 2015), $N$-acetylaspartate and creatine levels (Jung et al., 2005; Rae, Digney, McEwan, \& Bates, 2003), structural and functional connectivity (Hilger, Ekman, Fiebach, \& Basten, 2017; Li et al., 2009; van den Heuvel, Stam, Kahn, \& Hulshoff Pol, 2009), and cortical microstructure (Genc et al., 2018). Such relationships were identified for the overall brain as well as single brain regions (Jung \& Haier, 2007).

By contrast, the neural foundations of crystallized intelligence have been studied to a lesser degree. For example, 
Jung et al. (1999) conducted a magnetic resonance spectroscopy study, in which they targeted the left occipitoparietal white matter and employed the Wechsler Adult Intelligence Scale (WAIS) in order to obtain measures of verbal and performance IQ. Results showed a negative correlation between WAIS information subtest scores and choline levels as well as a positive correlation between vocabulary subtest scores and $N$-acetylaspartate levels. Pfleiderer et al. (2004) observed a positive correlation between vocabulary test performance and $\mathrm{N}$-acetylaspartate levels in the left dorsolateral prefrontal cortex and anterior cingulate cortex of healthy women but not men. In a large sample of over 200 individuals, Choi et al. (2008) investigated the relationship between cortical thickness and intelligence as measured by the verbal and performance IQ tests from the WAIS. The authors reported positive correlations between information subtest scores and cortical thickness in four different brain areas, namely, the anterior temporal cortex, the inferior temporal cortex, the opercular temporal cortex, and the medial temporal cortex. However, cortical thickness in the lateral parietal cortex was negatively associated with the information subtest. Vocabulary subtest scores were positively associated with cortical thickness in the anterior and medial temporal cortices and showed a negative association with cortical thickness in the lateral parietal cortex. Colom et al. (2009) investigated the neural correlates of fluid and crystallized intelligence in a large sample of 100 healthy individuals by means of voxelbased morphometry. In order to assess crystallized intelligence, they utilized a test battery consisting of verbal and numerical reasoning tests as well as a vocabulary test. Clusters in which regional grey matter was associated with crystallized intelligence also exhibited correlations between regional grey matter and fluid intelligence. These clusters were highly consistent with loci proposed by the parieto-frontal integration theory of intelligence (Jung \& Haier, 2007). The anterior temporal cortex, also known as Brodmann area 38, was the only region in which grey matter volume was uniquely correlated with crystallized intelligence. Using the same test battery, Roman et al. (2014) analysed the relationship between test results and three morphometric indices, namely, grey matter volume, cortical surface area, and cortical thickness, in over 100 individuals. Crystallized intelligence was associated with grey matter volume in the right frontal lobe, with cortical surface area in the frontal lobes of both hemispheres and with cortical thickness in the left parietal lobe. Using diffusion tensor imaging (DTI), Martinez et al. (2017) reconstructed the white matter connectomes of 80 healthy individuals. They found the number of interhemispheric connections as well as the ratio between interhemispheric and intrahemispheric connections to be positively correlated with verbal ability. These results were independent of sex or brain size.

It is noteworthy that most of the aforementioned studies quantified crystallized intelligence either by tests of verbal ability and vocabulary or by rather short information subtests included in larger intelligence test inventories such as the WAIS. Studies directly investigating the neural correlates of general knowledge as their only variable of interest are scarce. Usually, such studies tend to suffer from comparably small sample sizes or employ test inventories that have an inadequate number of items. For example, in a small clinical sample, Waltz et al. (1999) were able to show that semantic knowledge was lower in patients with anterior temporal lobe damage compared to patients with prefrontal cortex lesions or healthy controls. These findings are in accordance with the results reported by Colom et al. (2009), indicating that Brodmann area 38 plays a crucial rule for crystallized intelligence and semantic knowledge. Nevertheless, it is reasonable to say that the neural properties associated with general knowledge in healthy individuals remain largely unknown.

In order to close this gap of knowledge, the present study addresses the yet unanswered question whether interindividual differences in the brain's structure and function are linked to interindividual differences in general knowledge. It is well accepted that information constituting general knowledge is stored in synaptic connections (Wiltgen, Brown, Talton, \& Silva, 2004). Because individuals with more cortical grey matter volume hold more neurons (Leuba \& Kraftsik, 1994; Pakkenberg \& Gundersen, 1997) and cortical neuron number correlates with synapse amount (Karbowski, 2007), we hypothesized that individuals with more cortical grey matter volume should have a higher capacity for general knowledge. However, retrieving items of knowledge requires the ability to combine information from distributed memory (Wiltgen et al., 2004). The efficacy of retrieval is likely shaped by the properties of brain networks that can be estimated by means of probabilistic DTI fibre tractography or resting-state functional magnetic resonance imaging (fMRI). Thus, we assumed that individuals with greater cortical grey matter volume and more efficiently connected brain networks should hold more general knowledge.

We tested these hypotheses by calculating partial correlation coefficients between behavioural data (general knowledge and matrix reasoning test scores) and imaging data (estimates of brain volume and brain connectivity). Previous research has shown that male and female individuals differ with regard to certain brain properties such as cortical thickness, cortical surface area, and cortical grey matter volume, even after considering sex differences in body size (Escorial et al., 2015). Hence, we included sex and age as controlling variable in all analyses. Analyses were carried out at the level of the whole brain as well as for single brain regions. Correction for multiple comparisons was applied accordingly. Moreover, we performed multiple regression analysis with general knowledge or matrix reasoning test scores being regressed on age, sex, and the four brain properties, thereby controlling for confounding effects within potential structure-function relationships. In doing so, this study is able to deliver first results specifically targeted on the neural architecture of general knowledge and shed light on its relation to the biological basis of fluid intelligence.

\section{METHODS}

\section{Participants}

Three hundred thirty-two participants (172 men) aged between 18 and 75 years $(M=28.11, S D=10.74)$ took part in 
the study. The complete dataset was checked for extreme outliers as defined by Tukey's fences, that is, observations three interquartile ranges below the first or above the third quartile, respectively (Tukey, 1977). In following this approach, we had to exclude eight participants because of extreme outliers being detected in their behavioural or neuroimaging data. Thus, all of the reported analyses were performed on data from 324 participants (170 men) between 18 and 75 years of age $(M=28.20, S D=10.82)$. Because this is the first study to directly investigate the neural correlates of general knowledge, it was not possible to estimate the necessary sample size a priori based on existing literature. Instead, we collected data from a reasonably large sample and computed the achieved power post hoc using G*Power (Faul, Erdfelder, Buchner, \& Lang, 2009). The analysis was based on the regression coefficient for $\mathrm{NET}_{\text {struc }}$ from the multiple regression model predicting general knowledge (Figure 5(A)) $\left(f^{2}=.19\right.$, $\alpha=.05$, six predictors, 324 participants) and yielded a power of .99 , thereby indicating sufficient sample size. Table 1 provides descriptive information on the overall sample as well as male and female subjects. We did not observe a significant sex difference with regard to age $(t(322)=0.99, p=.322$, $d=.11)$. Two hundred ninety-four participants were right handed, and the remaining 30 participants were left handed as measured by the Edinburgh Handedness Inventory (Oldfield, 1971). This ratio is representative of the human population (Raymond \& Pontier, 2004). All participants had normal or corrected-to-normal vision and hearing. They were either paid for their participation or received course credit. All participants were naive to the purpose of the study and had no former experience with any of the conducted general knowledge or fluid intelligence tests. Participants had no history of psychiatric or neurological disorders, as examined by a selfreport questionnaire, and matched the standard inclusion criteria for fMRI examinations. Each participant completed the behavioural tests and neuroimaging measurements described in the succeeding texts. The study was approved by the local ethics committee of the Faculty of Psychology at Ruhr University Bochum. All participants had to give their written informed consent and were treated in accordance with the Declaration of Helsinki.

Table 1. Descriptive information on the overall, male, and female sample

\begin{tabular}{lccr}
\hline & Overall & Men & \multicolumn{1}{c}{ Women } \\
\hline$N(\%)$ & $324(100.00)$ & $170(52.47)$ & $154(47.53)$ \\
Age & $28.20(10.82)$ & $28.76(10.98)$ & $27.57(10.63)$ \\
BOWIT & $148.56(42.17)$ & $162.98(41.43)$ & $132.66(37.05)$ \\
BOMAT & $15.81(4.12)$ & $16.24(4.19)$ & $15.34(4.00)$ \\
GMV & $491.66(52.06)$ & $516.63(49.25)$ & $464.09(39.81)$ \\
WMV & $459.74(56.78)$ & $490.00(49.69)$ & $426.32(44.03)$ \\
NET $_{\text {struc }}$ & $258.33(25.15)$ & $265.88(25.26)$ & $250.00(22.29)$ \\
NET $_{\text {func }}$ & $0.27(0.04)$ & $0.28(0.04)$ & $0.26(0.04)$ \\
\hline
\end{tabular}

Note: Mean values and standard deviations (in parentheses) of age, BOWIT and BOMAT test results, and magnetic resonance imaging measurements (GMV, cortical grey matter volume; WMV, white matter volume; $\mathrm{NET}_{\text {struc}}$, global efficiency of structural network; $\mathrm{NET}_{\text {func }}$, global efficiency of functional network). BOWIT, Bochumer Wissenstest; BOMAT, Bochumer Matrizentest.

\section{Acquisition and analysis of behavioural data}

General knowledge and fluid intelligence were assessed by the use of two paper-and-pencil tests. The tests were conducted in a group setting of up to six participants seated at individual tables in a quiet and well-lighted room.

\section{General knowledge assessment}

General knowledge was measured with a German inventory called 'Bochumer Wissenstest' (BOWIT) (Hossiep \& Schulte, 2008). The BOWIT inventory comprises two parallel forms (A and B) with 154 items each. Both forms include 11 different knowledge facets that can be grouped into two major knowledge domains according to their factor analytical structure (Hossiep \& Schulte, 2008). The first domain is denoted 'Humanities' and includes the seven knowledge facets Arts/Architecture, Language/Literature, Geography/Logistics, Economics/ Law Philosophy/Religion, History/Archaeology, and Civics/Politics. The second domain is denoted 'Sciences' and comprises the four knowledge facets Mathematics/ Physics, Biology/Chemistry, Technology/Electronics, and Nutrition/Health. Within one test form, each knowledge facet is represented by 14 multiple-choice items. In order to assess every individual's degree of general knowledge as precisely as possible, all participants had to complete both test forms resulting in a total number of 308 items. The knowledge facets assessed by the BOWIT inventory are very similar to general knowledge inventories used in other studies (Ackerman, 1996, 2000; Ackerman et al., 2001; Bratko, Butkovic, \& Chamorro-Premuzic, 2010; Lynn, Irwing, \& Cammock, 2002; Lynn, Ivanec, \& Zarevski, 2009; Lynn, Wilberg, \& Margraf-Stiksrud, 2004; Rolfhus \& Ackerman, 1996, 1999). The BOWIT inventory fulfils all important quality criteria regarding different measures of reliability and validity (Hossiep \& Schulte, 2008). The inventory's manual specifies that split-half reliability is .96 , Cronbach's $\alpha$ is .95 , test-retest reliability is .96 , and parallel-form reliability between A and $\mathrm{B}$ is .91. Furthermore, convergent and discriminant validity are given for both test forms because they are strongly correlated with the general knowledge module of a well-established German intelligence test $(r>.70$, I-S-T 2000R) (Liepmann, Beauducel, Brocke, \& Amthauer, 2007) and not associated with tests of perceptual speed $(r<.15$, ZVT) (Oswald \& Roth, 1987). BOWIT test performance is significantly correlated with German high school GPA ( $r=-.28$, reverse coded) and gross salary per year for professionals $(r=.21-.30)$ (Hossiep \& Schulte, 2008), demonstrating its predictive validity. The norm sample consists of about 2300 individuals from throughout Germany. The age range is between 18 and 66 years with men and women being represented equally. Because the vast majority of individuals from the norm sample received an academic education, the BOWIT inventory can be considered a highly suitable test instrument for samples mainly composed of university students and other individuals that are expected to have above average general knowledge. 


\section{Fluid intelligence assessment}

Fluid intelligence was measured with a German matrix reasoning test called 'Bochumer Matrizentest' (BOMAT) (Hossiep, Hasella, \& Turck, 2001), which is widely used in neuroscientific research (Jaeggi, Buschkuehl, Jonides, \& Perrig, 2008; Klingberg, 2010; Oelhafen et al., 2013; Takeuchi et al., 2014). The test examines non-verbal mental abilities that contribute to fluid intelligence and is similar to Raven's Advanced Progressive Matrices (Raven, 1990). We conducted the 'advanced short version' of the BOMAT, which has the advantage of high discriminatory power in samples with generally high intellectual abilities, thus avoiding possible ceiling effects (Jaeggi et al., 2008). The BOMAT inventory comprises two parallel test forms (A and B) with 29 matrix reasoning items each. Because the focus of the present study was not on fluid intelligence and administering both test forms is rather time consuming, participants had to complete only one of the two forms being randomly assigned to them. The BOMAT also fulfils the necessary test quality criteria regarding different reliability and validity measures. Split-half reliability is .89 , Cronbach's $\alpha$ is .92, and parallel-form reliability between A and B is .86 (Hossiep et al., 2001). Additionally, convergent and predictive validity are given for both BOMAT test forms because they are strongly correlated with other fluid intelligence inventories $(r=.59)$, tests of perceptual speed $(r=.51)$ and German high school GPA ( $r=-.35)$ (Hossiep et al., 2001). The recent norm sample consists of about 2100 individuals with an age range between 18 and 60 years and both sexes being represented equally.

\section{General knowledge and fluid intelligence analysis}

The overall test performance for the BOWIT inventory was computed as a sum score of all 308 items from both test forms A and B. Test performance for the BOMAT inventory was computed as a sum score of the last 28 items. The first item was disregarded because of its low difficulty as recommended by the test manual.

\section{Acquisition of imaging data}

All imaging data were acquired at the Bergmannsheil Hospital in Bochum, Germany, using a 3T Philips Achieva scanner with a 32-channel head coil.

\section{Anatomical imaging}

For the purpose of segmenting brain scans into grey and white matter sections as well as for the identification of anatomical landmarks for connectivity analyses, a T1-weighted high-resolution anatomical image was acquired (MP-RAGE, $\mathrm{TR}=8.2$ milliseconds, $\mathrm{TE}=3.7$ milliseconds, flip angle $=8^{\circ}$, 220 slices, matrix size $=240 \times 240$, resolution $=1 \times 1 \times 1 \mathrm{~mm}$ ). The acquisition time of the anatomical image was 6 minutes.

\section{Diffusion-weighted imaging}

For the analysis of structural network connectivity, diffusionweighted images were acquired using echo planar imaging $(\mathrm{TR}=7652$ milliseconds, $\mathrm{TE}=87$ milliseconds, flip angle $=90^{\circ}, 60$ slices, matrix size $=112 \times 112$, resolution $=2 \times 2 \times 2 \mathrm{~mm}$ ). Diffusion weighting was isotropically distributed along 60 directions using a $b$-value of 1000 seconds $/ \mathrm{mm}^{2}$. Additionally, six volumes with no diffusion weighting ( $b=0$ seconds $/ \mathrm{mm}^{2}$ ) were acquired as an anatomical reference for motion correction and for computation of diffusion coefficients during the diffusion sequence. In order to increase the signal-to-noise ratio, we acquired three consecutive scans that were subsequently averaged (Genç, Bergmann, Singer, \& Kohler, 2011; Genç et al., 2011). The total acquisition time of diffusion-weighted images was 30 minutes.

\section{Resting-state imaging}

For the analysis of functional network connectivity, fMRI resting-state images were acquired using echo planar imaging $(\mathrm{TR}=2000$ milliseconds, $\mathrm{TE}=30$ milliseconds, flip angle $=90^{\circ}, 37$ slices, matrix size $=80 \times 80$, resolution $=3 \times 3 \times 3 \mathrm{~mm})$. The acquisition time of resting-state images was 7 minutes.

\section{Analysis of imaging data}

\section{Analysis of anatomical data}

We used published surface-based methods in FREESURFER (http://surfer.nmr.mgh.harvard.edu, version 5.3.0) to reconstruct the cortical surfaces of the T1-weighted images. The details of this procedure have been described elsewhere (Dale, Fischl, \& Sereno, 1999; Fischl, Sereno, \& Dale, 1999). The automatic reconstruction steps included skull stripping, grey and white matter segmentation (Figure 2, left), and reconstruction and inflation of the cortical surface. These preprocessing steps were performed for each participant individually. Subsequently, each individual segmentation was quality controlled slice by slice, and inaccuracies for the automatic steps were corrected by manual editing if necessary. We selected a set of 42 brain regions per hemisphere to be examined with regard to relationships between specific brain properties and cognitive abilities (Figure 2, left). These regions were extracted by an automatic segmentation procedure in FREESURFER. Thirty-four out of 42 regions were extracted as cortical grey matter regions following a gyral/sulcal-based parcellation procedure on the reconstructed cortical surface (Desikan et al., 2006). The remaining eight regions were extracted as subcortical regions (Fischl et al., 2004). In addition, white matter was also segmented into 34 distinct regions per hemisphere. Hereto, each white matter voxel was labelled to the nearest cortical grey matter voxel within a distance limit of $5 \mathrm{~mm}$. This resulted in 34 white matter regions corresponding to the 34 gyral-labelled grey matter regions (Klein et al., 2014; Salat et al., 2009). Finally, six regions representing the four ventricles of the brain were extracted to serve as a reference for later BOLD signal analyses. Brain segmentation yielded an estimate of the overall cortical grey matter volume (GMV) and the overall white matter volume (WMV). Furthermore, the grey matter volume of each individual cortical and subcortical region was assessed. In a final step, the cortical, subcortical, and ventricle regions were linearly transformed into the native space of the diffusion-weighted and restingstate images (Figure 2, middle). The transformed regions 


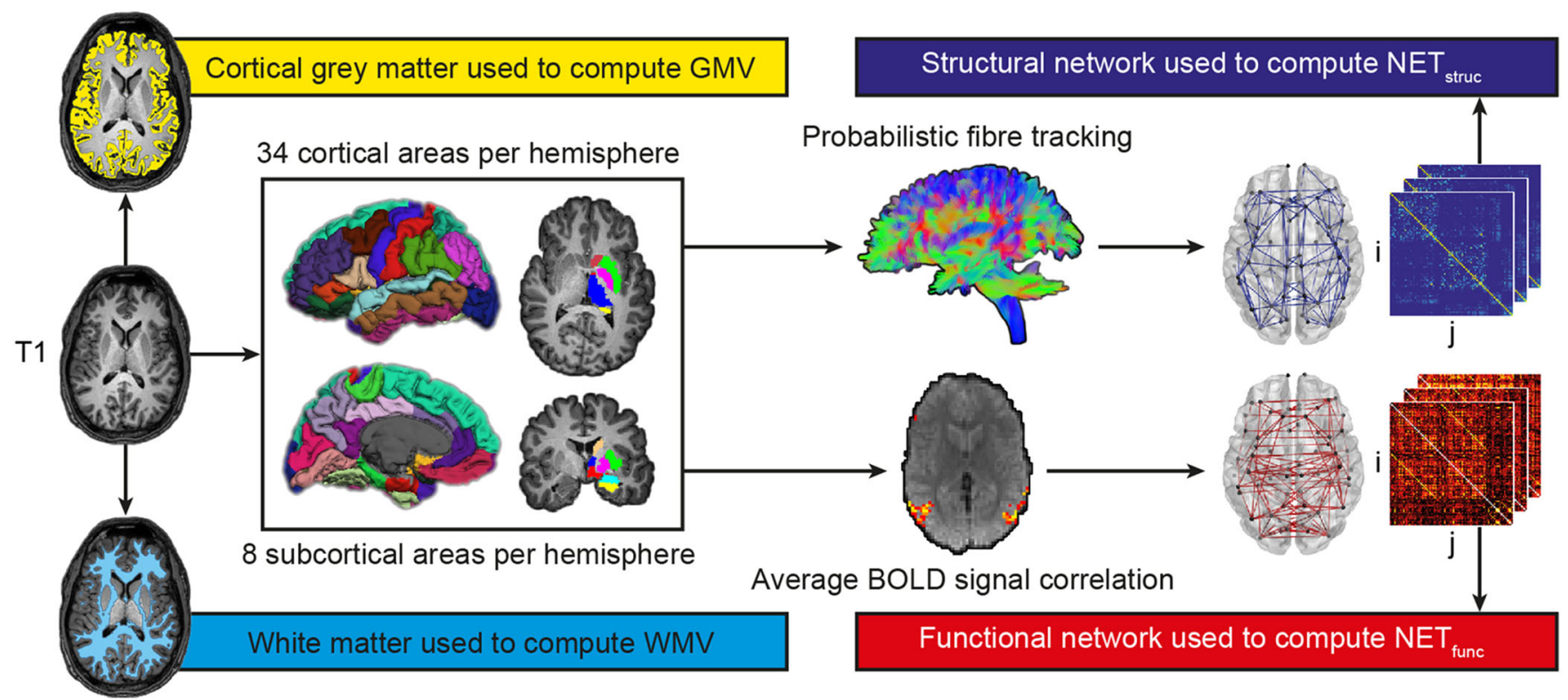

1. Brain segmentation (Desikan-Killiany)

2. Network definition, pruning and analysis

Figure 2. Processing steps for the estimation of brain properties. First, T1-weighted anatomical images were partitioned into segments of cortical grey matter and white matter of which respective overall volume estimates (GMV, cortical grey matter volume; WMV, white matter volume) were computed. Second, T1weighted images were segmented into 34 cortical and eight subcortical brain regions per hemisphere according to the Desikan-Killiany atlas. Third, the respective masks were linearly transformed into the native space of the diffusion-weighted and resting-state images. For the diffusion-weighted images, probabilistic fibre tracking was performed with the brain masks serving as seed and target regions. For the resting-state images, partial correlations between average BOLD time courses of all brain regions were computed. Fourth, structural and functional brain networks were reconstructed with edges being weighted by the results of either probabilistic fibre tractography (streamline count) or BOLD signal correlation analysis. Finally, global efficiency was computed as a measure of structural $\left(\mathrm{NET}_{\text {struc }}\right)$ and functional network connectivity $\left(\mathrm{NET}_{\text {func }}\right)$.

served as network nodes for graph theoretical connectivity analyses (Figure 2, right).

\section{Analysis of diffusion data}

Diffusion tensor modelling and probabilistic fibre tractography were carried out with FMRIB's Diffusion Toolbox as implemented in FSL version 5.0.7. The details of this procedure have been described elsewhere (T. E. Behrens et al., 2003). Preprocessing steps included a correction for eddy currents (eddy_correct) and head motion as well as a correction of the gradient direction for each volume using the rotation parameters that emerged from head motion. As described earlier, the 42 cortical and subcortical regions from each hemisphere were transformed into the native space of the diffusion-weighted images to serve as seed and target regions for probabilistic fibre tractography. We used a dual-fibre model as implemented in the latest version of BEDPOSTX. The model allows for the representation of two fibre orientations per voxel when more than one orientation is supported by the data. This enables modelling of crossing fibres and produces more reliable results compared with single-fibre models (T. E. J. Behrens, Berg, Jbabdi, Rushworth, \& Woolrich, 2007). Probabilistic fibre tractography was carried out using the classification targets approach implemented in FMRIB's Diffusion Toolbox (T. E. Behrens et al., 2003; M. X. Cohen, Schoene-Bake, Elger, \& Weber, 2009). At each voxel, 5000 tract-following samples were generated with a step length of $0.5 \mathrm{~mm}$ and a curvature threshold of 0.2 (only allowing for angles larger than $80^{\circ}$ ). The connectivity between a seed voxel and a specific target region was quantified by the number of streamlines originating in the seed voxel and reaching the respective target region. The overall connectivity between two brain regions was computed as the sum of all streamlines proceeding from the seed to the target region and vice versa.

\section{Analysis of resting-state data}

Resting-state data were preprocessed using MELODIC, which is also a part of the FSL toolbox. Images were preprocessed in a number of steps: discarding the first two epipolar plane image volumes from each resting-state scan to allow for signal equilibration, motion and slice-timing correction, high-pass temporal frequency filtering $(0.005 \mathrm{~Hz})$. Spatial smoothing was not applied in order to avoid the introduction of spurious correlations in neighbouring voxels. Analogous to the analysis of the diffusion data, all 42 subcortical and cortical regions from each hemisphere were transformed into the native space of the resting-state images to serve as seed and target regions for functional connectivity analysis. For each region, we calculated a mean resting-state time course by averaging the preprocessed time courses of corresponding voxels. We computed partial correlations between the average time courses of all cortical and subcortical regions, while controlling for several nuisance variables. We regressed out the trajectories of all six motion parameters as well as the mean time courses averaged across all voxels representing white matter or cerebrospinal fluid (Genc, Scholvinck, Bergmann, Singer, \& Kohler, 2015). 


\section{Graph theoretical analysis of imaging data}

For the purpose of graph theoretical connectivity analyses, we defined a brain network with 84 nodes, consisting of the 68 cortical and 16 subcortical regions mentioned earlier, which yielded a total of 3486 edges. Network edges were weighted in two different ways. In the structural brain network, each edge weight represented the total number of streamlines between two brain regions. In the functional brain network, each edge weight represented the partial correlation between BOLD signal time courses of two brain regions. In the case of negative partial correlation coefficients, we used absolute values as edge weights.

\section{Graph metrics}

Graph metrics were computed with the Brain Connectivity Toolbox (Rubinov \& Sporns, 2010) along with in-house MATLAB code. Both of the weighting methods described earlier resulted in a symmetrical adjacency matrix with 84 nodes. In order to remove spurious network connections, we employed Holm-Bonferroni pruning with a threshold of $0(\alpha=.01$, one tailed) as proposed by Ivkovic, Kuceyeski, and Raj (2012). In doing so, no edges were removed from the functional network, while 2540 edges were removed from the structural network. Among the 946 edges remaining in the structural network, we observed considerably more intrahemispheric connections $(573,60.57 \%)$ than interhemispheric connections $(373,39.43 \%)$. We chose to quantify network connectivity by means of global efficiency because this graph metric has been used in previous studies investigating the association between brain network properties and cognitive abilities (Fischer, Wolf, Scheurich, \& Fellgiebel, 2014; Hilger et al., 2017; Li et al., 2009; Ryman et al., 2014; Ryman et al., 2016; van den Heuvel et al., 2009). Global efficiency provides information about how efficiently information can be exchanged within a network (Latora \& Marchiori, 2001). Higher edge weights and shorter path lengths will typically increase this metric. The shortest path lengths between all pairs of nodes are contained in a distance matrix $d$. This matrix is obtained by calculating the inverse of the weighted adjacency matrix and running a search algorithm like Dijkstra's algorithm (Dijkstra, 1959) used in the Brain Connectivity Toolbox. Global efficiency of a specific brain region $i\left(E_{i}\right)$, also called nodal efficiency, is calculated as the average inverse shortest path length between that particular node $i$ and all other nodes $j$ within the network $G$, with $i$ unequal $j$. The overall global efficiency $(E)$ is calculated in a similar fashion as the average inverse shortest path length between each pair of nodes within the whole network $G$ :

$$
E=\frac{1}{n} \sum_{i \in G} E_{i}=\frac{1}{n} \sum_{i \in G} \frac{\sum_{j \in G, j \neq i} d_{i j}^{-1}}{n-1}
$$

\section{Statistical analysis}

Statistical analyses were performed using MATLAB version R2017b (The MathWorks Inc., Natick, MA) and SPSS version 25 (SPSS Inc., Chicago, IL). For all analyses, linear parametric methods were used. Testing was two tailed with an $\alpha$-level of .05 that was Bonferroni corrected for multiple comparisons if necessary.

Analysis of sex differences

We analysed our behavioural and imaging data in view of potential sex differences. To this end, we compared male and female subjects with regard to overall test performance in the BOWIT and BOMAT inventories, test performance in the single knowledge facets of the BOWIT inventory, and all four brain properties GMV, WMV, $\mathrm{NET}_{\text {struc }}$, and $\mathrm{NET}_{\text {func }}$ (Figure $\mathrm{S} 1$ ). Statistically significant differences were revealed by two-sample $t$-tests and quantified as effect sizes in the form of Cohen's $d$ (J. Cohen, 1992).

\section{Partial correlation analysis}

We examined structure-function relationships on a wholebrain level and correlated general knowledge as well as fluid intelligence with each of the four brain properties included in this study, while partialling out the effects of age and sex (Figure 3). In order to account for multiple comparisons, we applied Bonferroni correction with a factor of four $(\alpha=.05 /$ $4=.0125$ ) due to the four brain properties that were analysed. Subsequently, we computed the same partial correlations on the level of single brain regions. To this end, we averaged the four brain properties of each brain region across both hemispheres resulting in 42 regions for GMV, $\mathrm{NET}_{\text {struc }}$, and $\mathrm{NET}_{\text {func }}$ and 34 regions for WMV (no subcortical regions available). Therefore, the $\alpha$-level of each partial correlation coefficient was Bonferroni corrected with a factor of 42 $(\alpha=.05 / 42=.0012, \alpha=.01 / 42=.0002$; Figure 4). Additionally, we computed partial correlation coefficients between general knowledge, fluid intelligence, and all four brain properties with age and sex as controlling variables (Table 2).

\section{Multiple regression analysis}

To examine the aforementioned associations with regard to the unique contribution of each variable included in the partial correlation analyses, we utilized multiple regression analysis. In our first model, general knowledge was regressed on the four brain properties GMV, WMV, $\mathrm{NET}_{\text {struc }}$, and $\mathrm{NET}_{\text {func }}$ as well as age and sex (Figure 5(A)). In our second model, fluid intelligence served as the dependent variable, while the four brain properties GMV, WMV, $\mathrm{NET}_{\text {struc }}$, and $\mathrm{NET}_{\text {func }}$ as well as age and sex served as independent variables (Figure 5(B)). In order to investigate the role of sex more specifically, we reanalysed both models for men (Figure S2(A) and (B)) and women (Figure S2(C) and (D)) separately. Hence, sex was excluded as an independent variable and used as a group variable. Given the significant relationship between the BOMAT and BOWIT test scores, we computed another version of the first model with fluid intelligence serving as an additional predictor of general knowledge (Figure S3).

The analyses were not preregistered. An SPSS file containing preprocessed data and MATLAB scripts used for various aspects of data analysis can be obtained from an OSF page (https://osf.io/zmw5y/). The raw data used for the analyses are part of an ongoing research project and can only be obtained upon reasonable request. They will be made publicly available after data acquisition is completed. 

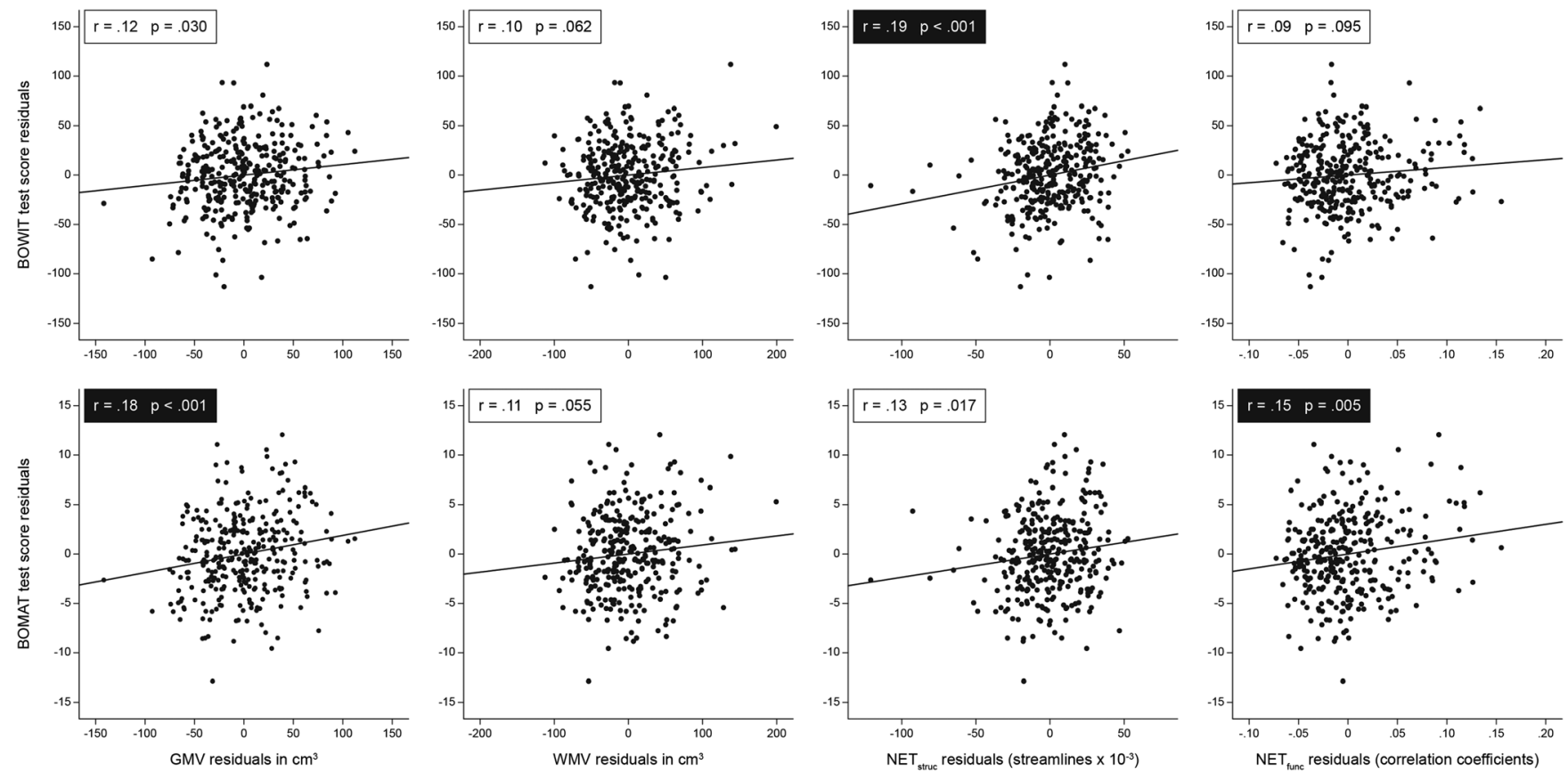

Figure 3. Partial correlation analysis of associations at the whole-brain level. Scatter plots illustrating the relationships between brain properties (GMV, cortical grey matter volume; WMV, white matter volume; $\mathrm{NET}_{\text {struc }}$, global efficiency of structural network derived from probabilistic fibre tracking; $\mathrm{NET}_{\text {func }}$, global efficiency of functional network derived from BOLD signal correlations) and cognitive abilities (general knowledge and fluid intelligence). All associations are controlled for the effects of age and sex. The $\alpha$-level was corrected for multiple comparisons using the Bonferroni method and a factor of four $(\alpha=.05 /$ $4=.0125)$ due to the four brain parameters under investigation. Statistically significant correlation coefficients surviving Bonferroni correction $(N=324$, $p<.0125)$ are highlighted with black boxes. BOWIT, Bochumer Wissenstest; BOMAT, Bochumer Matrizentest.

\section{RESULTS}

\section{Associations at the whole-brain level}

Descriptive information including mean test scores and brain properties measured at the whole-brain level are presented in Table 1. We observed significant associations for most of the brain properties included in the study (Figure 3). As presumed, general knowledge was positively correlated with $\mathrm{NET}_{\text {struc }}(r=.19, p<.001,95 \%$ confidence interval, CI $[.08, .29])$, indicating that individuals with more efficiently connected fibre networks have a higher level of general knowledge. The association between general knowledge and GMV failed to reach statistical significance after Bonferroni correction was applied $(r=.12, p=.030,95 \%$ CI $[.01, .23])$. Further, general knowledge was not significantly associated with WMV $(r=.10, p=.062,95 \%$ CI $[-.01, .21])$ and $\mathrm{NET}_{\text {func }}$ $(r=.09, p=.095,95 \%$ CI $[-.02, .20])$. As expected, fluid intelligence was positively correlated with GMV $(r=.18$, $p<.001,95 \%$ CI $[.07, .28])$ and $\operatorname{NET}_{\text {func }}(r=.15$, $p=.005,95 \%$ CI $[.04, .25])$. However, we observed no significant correlation between fluid intelligence and WMV $(r=.11, p=.055,95 \%$ CI $[.00, .22])$, and the association between fluid intelligence and $\mathrm{NET}_{\text {struc }}$ lost its statistical significance after we accounted for multiple comparisons $(r=.13, p=.017,95 \%$ CI $[.02, .24])$. Importantly, all of the aforementioned associations were controlled for the effects of age and sex.

\section{Associations at the level of single brain regions}

In a next step, we refined our analyses in order to identify specific brain regions showing exceptionally high structure-function associations, which might provoke the correlations observed at the whole-brain level. To this end, we defined 34 cortical and eight subcortical brain regions per hemisphere (Desikan et al., 2006; Fischl et al., 2004; Salat et al., 2009). For all of these 42 brain regions, we correlated each brain property with both general knowledge and fluid intelligence, while correcting for multiple comparisons and controlling for the effects of age and sex (Figure 4).

General knowledge was significantly associated with GMV in the pars opercularis $(r=.20, p<.001,95 \% \mathrm{CI}$ $[.09, .30])$ and with WMV in the pars opercularis $(r=.20$, $p<.001,95 \%$ CI $[.09, .30])$. Furthermore, 11 brain regions showed significant positive correlations between general knowledge and $\mathrm{NET}_{\text {struc }}(r=.18-.23, p<.001,95 \% \mathrm{CI}$ $[.07, .28]-[.12, .33])$. Respective regions were distributed across the whole brain with no clear pattern of clustering in any of the lobes. Corresponding to the results found at the whole-brain level, general knowledge was not significantly correlated with $\mathrm{NET}_{\text {func }}$ in any of the brain regions. Fluid intelligence was significantly associated with GMV in the parahippocampal gyrus $(r=.18, p<.001,95 \%$ CI [.07, $.28])$, the pars triangularis $(r=.22, p<.001,95 \%$ CI [.11, $.32])$, the rostral anterior cingulate cortex $(r=.18$, $p<.001,95 \%$ CI $[.07, .28])$, and the amygdala $(r=.18$, $p<.001,95 \%$ CI $[.07, .28])$. Furthermore, we observed a 

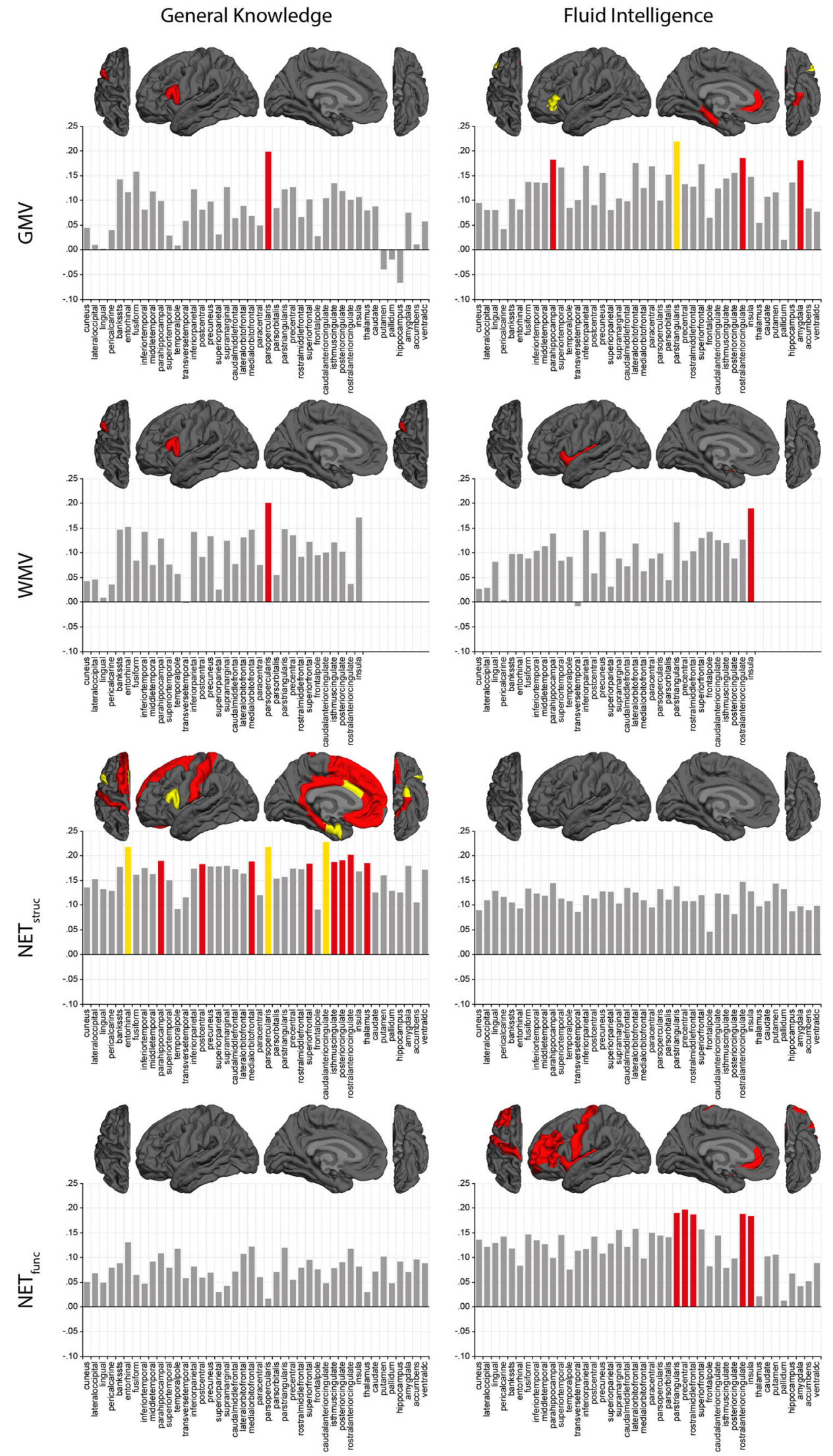

Figure 4. Partial correlation analysis of associations at the level of single brain regions. Brain properties from each brain region (GMV, cortical grey matter volume; WMV, white matter volume; $\mathrm{NET}_{\text {struc }}$, global efficiency of structural network; $\mathrm{NET}_{\text {func, }}$, global efficiency of functional network) were averaged across both hemispheres and correlated with general knowledge (left column) and fluid intelligence (right column). Confounding effects of age and sex were partialled out. Bonferroni correction was applied with a factor of 42 to account for multiple comparisons $(\alpha=.05 / 42=.0012 ; \alpha=.01 / 42=.0002)$. Correlations that reached statistical significance after Bonferroni correction are highlighted by red $(p<.0012)$ or yellow $(p<.0002)$ bars. Likewise, respective brain regions are colour coded on a cortical surface. 
Table 2. Partial correlations among cognitive abilities and brain properties

\begin{tabular}{llllll}
\hline & BOWIT & BOMAT & GMV & WMV & NET $_{\text {struc }}$ \\
\hline BOMAT & $.25^{* *}$ & - & - & - & - \\
GMV & $.12^{*}$ & $.18^{* *}$ & - & - & - \\
WMV & .10 & .11 & $.68^{* *}$ & - & - \\
NET $_{\text {struc }}$ & $.19^{* *}$ & $.13^{*}$ & $.66^{* *}$ & $.40^{* *}$ & - \\
NET $_{\text {func }}$ & .09 & $.15^{* *}$ & .08 & .08 & .05 \\
\hline
\end{tabular}

Note: Age and sex are used as controlling variables. $N=324$. BOWIT, Bochumer Wissenstest; BOMAT, Bochumer Matrizentest; GMV, cortical grey matter volume; WMV, white matter volume; $\mathrm{NET}_{\text {struc }}$, global efficiency of structural network; $\mathrm{NET}_{\text {func }}$, global efficiency of functional network. $* p<.05$ (two tailed). $* * p<.01$ (two tailed).

significant positive correlation between fluid intelligence and WMV in the insula $(r=.19, p<.001,95 \%$ CI $[.08, .29])$. We did not observe any significant associations between fluid intelligence and $\mathrm{NET}_{\text {struc }}$, but fluid intelligence was positively correlated to $\mathrm{NET}_{\text {func }}$ in five brain regions, namely, the pars triangularis $(r=.19, p<.001,95 \%$ CI $[.08, .29])$, the precentral gyrus $(r=.20, p<.001,95 \%$ CI $[.09, .30])$, the rostral middle frontal gyrus $(r=.19, p<.001,95 \% \mathrm{CI}$ $[.08, .29])$, the rostral anterior cingulate cortex $(r=.19$, $p<.001,95 \%$ CI $[.08, .29])$, and the insula $(r=.18$, $p<.001,95 \%$ CI $[.07, .28])$.

\section{Predicting general knowledge and fluid intelligence by means of multiple regression analysis}

In accordance with previous research (Ackerman et al., 2001), our data showed a positive correlation between general knowledge and age $(r=.48, p<.001,95 \%$ CI [.39, $.56])$ as well as significant sex differences with men performing better than women for the majority of knowledge facets in the BOWIT $(t(322)=6.92, p<.001)$ (Figure S1(A)). Importantly, many of the brain properties included in this study are also associated with age (e.g. GMV, $r=-.40, p<.001,95 \% \mathrm{CI}[-.49,-.30])$ and show sex differences with male subjects having a higher brain volume $(\mathrm{GMV}, t(322)=10.60, p<.001 ; \mathrm{WMV}, t(322)=12.16$, $p<.001)$ and more efficiently connected brain networks $\left(\mathrm{NET}_{\text {struc }}, t(322)=5.97, p<.001 ; \mathrm{NET}_{\text {func }}, t(322)=3.81\right.$, $p<.001$ ) than female subjects (Figure S1(B)). Furthermore, many of the brain properties are significantly correlated with each other, even after controlling for the effects of age and sex (Table 2). Thus, it is reasonable to assume that the observed correlations between brain properties and cognitive test performance might be confounded by age, sex, or some of the explained variance being shared by multiple brain properties. In order to address these issues, we employed an approach similar to recent studies investigating the neural foundation of fluid intelligence (Kievit et al., 2012; Ritchie et al., 2015). By means of multiple regression analysis, we were able to extract the unique contribution of each brain property in predicting general knowledge and fluid intelligence.

In our first model, general knowledge was regressed on age, sex, and the four brain properties included in the whole-brain analyses mentioned earlier (Figure 5(A)). The analysis yielded strong and highly significant associations between age and general knowledge $(\beta=.49, p<.001)$ as well as sex and general knowledge $(\beta=-.26, p<.001)$ with older and male individuals performing better on the BOWIT. In accordance with partial correlation analyses, general knowledge was not significantly associated with GMV $(\beta=-.07, p=.517)$, WMV $(\beta=.06, p=.449)$, or $\mathrm{NET}_{\text {func }}$ $(\beta=.07, p=.131)$. Importantly, $\mathrm{NET}_{\text {struc }}$ remained as the only brain property showing a unique contribution in predicting general knowledge $(\beta=.19, p=.006)$. Overall, this regression model was able to explain $36.80 \%$ of variance in general knowledge.

In our second model, fluid intelligence was regressed on age, sex, and the four brain properties (Figure 5(B)). Again, we observed results that were in accordance with those obtained by partial correlation analyses. The only brain properties showing unique contributions in predicting fluid intelligence were GMV $(\beta=.24, p=.042)$ and $\mathrm{NET}_{\text {func }}$
A

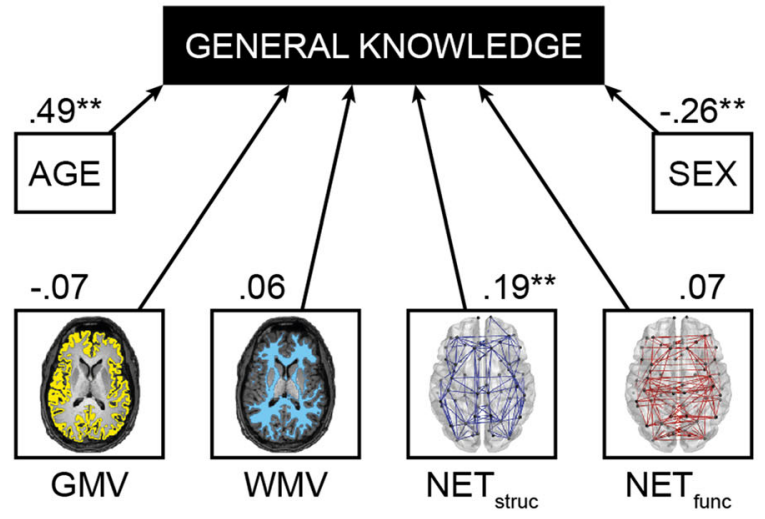

B

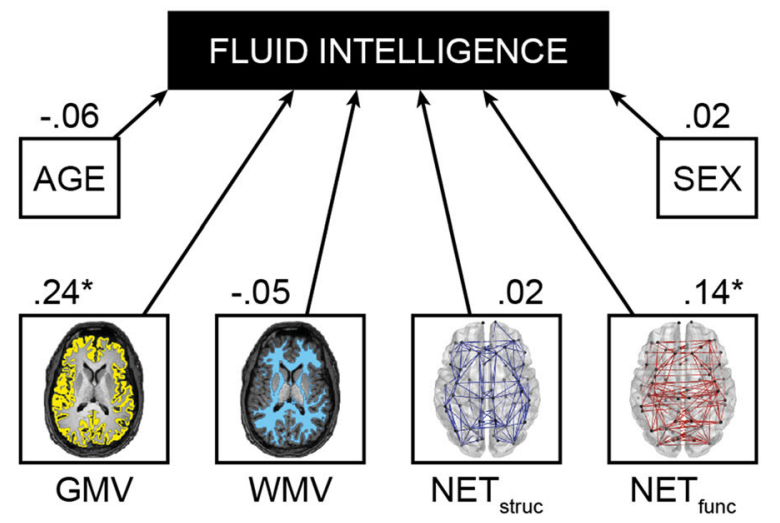

Figure 5. Results of multiple regression analysis. (A) General knowledge was regressed on age, sex, and four brain properties (GMV, cortical grey matter volume; WMV, white matter volume; $\mathrm{NET}_{\text {struc }}$, global efficiency of structural network; $\mathrm{NET}_{\text {func, }}$ global efficiency of functional network). (B) Accordingly, fluid intelligence was regressed on age, sex, and four brain properties. Standardised regression coefficients are depicted next to the respective arrows. $p<.05$; $p<.01$. 
$(\beta=.14, p=.010)$. The remaining brain properties WMV $(\beta=-.05, p=.609)$ and $\operatorname{NET}_{\text {struc }}(\beta=.02, p=.824)$ as well as age $(\beta=-.06, p=.420)$ and sex $(\beta=.02, p=.764)$ were not significantly associated with fluid intelligence. This model explained $9.66 \%$ of variance in fluid intelligence.

Given the substantial sex differences in terms of BOWIT test scores and brain properties, we decided to compute the aforementioned multiple regression models for both sexes separately (Figure S2). We included the same variables as described for the first and second model but used sex as a group variable instead of an independent variable. When regressing general knowledge on age and the four brain properties, we observed the same pattern of results for both sexes (Figure S2(A) and (C)). As with the multiple regression analysis for the overall sample, age was significantly associated with general knowledge in men $(\beta=.58, p<.001)$ and women $(\beta=.47, p<.001)$. The association between $\mathrm{NET}_{\text {struc }}$ and general knowledge, which we found to be statistically significant in the overall sample, could also be observed for the male subgroup $(\beta=.19, p=.042)$ and the female subgroup $(\beta=.17, p=.096)$. Both regression coefficients were of the same magnitude but only reached statistical significance at the level of $\alpha<.1$ for female subjects. This might be explained by the substantial decrease in sample size that is inevitable when analysing subgroups. When computing the same regression model with fluid intelligence serving as the dependent variable, we found the results from the analysis of the overall sample to be a combination of different results from the male and female subgroups (Figure S2(B) and (D)). In men, GMV exhibited the only significant association with fluid intelligence $(\beta=.35, p=.018)$, and in women, $\mathrm{NET}_{\text {func }}$ turned out to be the only significant predictor $(\beta=.19, p=.020)$.

Our data showed a significant correlation between general knowledge and fluid intelligence $(r=.16, p=.005$, $95 \%$ CI $[.05, .26])$. This is in line with the assumptions made by cognitive investment theory, namely, that the acquisition of general knowledge is dependent on an individual's fluid intelligence (Ackerman, 1996; Horn \& Cattell, 1967; Kvist \& Gustafsson, 2008). We took this into consideration by computing a slightly different version of the first model that included fluid intelligence as an additional predictor of general knowledge (Figure S3). This model was able to predict slightly more variance $\left(R^{2}=.40\right)$ because fluid intelligence turned out to be an independent predictor of general knowledge $(\beta=.19, p<.001)$. Apart from that, this alternative model did not result in any substantial changes regarding the other regression coefficients.

\section{DISCUSSION}

The overarching goal of the current study was to identify the neural correlates of interindividual differences in general knowledge. However, we also examined the biological foundations of fluid intelligence in order to revisit previously reported evidence. The respective findings concerning fluid intelligence shall be discussed briefly before turning towards general knowledge.
At the whole-brain level, fluid intelligence was significantly associated with GMV and $\mathrm{NET}_{\text {func. These results are }}$ in line with previous research linking fluid intelligence with cortex volume (Narr et al., 2007; Pietschnig et al., 2015; Posthuma et al., 2002) and functional network connectivity (van den Heuvel et al., 2009). Interestingly, the association between fluid intelligence and WMV, which had been demonstrated in the past (Narr et al., 2007), failed to reach statistical significance by a narrow margin. Moreover, fluid intelligence was associated with $\mathrm{NET}_{\text {struc }}$, replicating previous results regarding structural network connectivity (Fischer et al., 2014; Li et al., 2009), but this relationship lost its statistical significance after accounting for multiple comparisons. When employing multiple regression analysis, GMV and $\mathrm{NET}_{\text {func }}$ still remained as the only brain properties showing significant associations with fluid intelligence, which is comparable with results from previous studies (Ritchie et al., 2015; van den Heuvel et al., 2009). Computing the multiple regression analysis for both sexes separately led to different results for male and female subjects. In the male subgroup, we observed an association between GMV and fluid intelligence, while $\mathrm{NET}_{\text {func }}$ remained as the only significant predictor of fluid intelligence in the female subgroup. On the population level, men and women do not show substantial differences regarding fluid intelligence, but our results might indicate that both sexes draw on different aspects of their neural substrate in order to achieve comparable levels of cognitive performance. Naturally, GMV is closely linked to cortical neuron count and should thus be representative of the potential computing power an individual has. $\mathrm{NET}_{\text {func }}$ might be an indicator of how well these processing units interact with each other. It is conceivable that intelligent thinking in men is more dependent on GMV and sheer neuron count because male brains might show less variation in effective communication between neurons or benefit less from it. Contrary to that, women might take substantial advantage of the way in which their cortical neurons interact with each other, while the mere number of neurons does not influence fluid intelligence remarkably. Because the relationship between GMV and fluid intelligence was only present in the male subgroup, our data are able to explain the absence of notable sex differences in fluid intelligence despite men on average having about $10 \%$ larger brains compared with women (Ruigrok et al., 2014). However, it has to be noted that our results do not correspond with those from a metaanalysis conducted by Pietschnig et al. (2015), which did not show any substantial sex differences in the association between brain size and intelligence. However, the authors acknowledge the fact that brain size is just one out many variables related to intelligence. They also state that 'many of these factors have effects on IQ that are incremental and compensatory to those of brain size, indicating that none of these factors seems to be necessary or sufficient for intelligence, with supervenience (many-to-one), not isomorphism (one-to-one), best describing their relationship'.

At the level of single brain regions, we found fluid intelligence to be associated with the neural properties of cortical areas that are also included as relevant anatomical structures in the parieto-frontal integration theory (Jung \& Haier, 
2007). For example, we observed a significant correlation between fluid intelligence and GMV in the pars triangularis. According to parieto-frontal integration theory, this structure plays a crucial role in generating various solutions to a given problem (Jung \& Haier, 2007). Moreover, the pars triangularis constitutes part of Broca's area and is involved in language processing. Language provides the possibility of inner speech and enables us to break down a complex problem into a series of sub-steps represented in language sentences (Varley, 2007). Thus, it might serve as an important cognitive tool even when dealing with abstract visuospatial problems as posed in matrix reasoning tests. Further, GMV was significantly associated with fluid intelligence in the rostral anterior cingulate cortex, an area that is believed to engage in response selection as well as the inhibition of competing responses. For the insular cortex, we observed a significant association between WMV and fluid intelligence. Originally, this area was not included in the parieto-frontal integration theory model by Jung and Haier (2007) but was recognized by a more recent meta-analysis (Basten, Hilger, $\&$ Fiebach, 2015). The analysis of $\mathrm{NET}_{\text {func }}$ is noteworthy because it was able to replicate previous findings on the relationship between intelligence and functional connectivity. Comparable with our approach, Hilger et al. (2017) employed graph theoretical measures to analyse fMRI resting-state data. Their results showed positive associations between intelligence and nodal resting-state efficiency computed for the anterior insula and the dorsal anterior cingulate cortex. This is in good accordance with our findings that include the insula and the rostral anterior cingulate cortex as well. Moreover, we also observed a significant association between fluid intelligence and $\mathrm{NET}_{\text {func }}$ of the pars triangularis. The fact that fluid intelligence was not only related to volumetric features of the pars triangularis, the rostral anterior cingulate cortex, and the insula but also correlated with the functional connectivity of these areas emphasizes the major role of these three brain regions in affecting cognitive performance.

In view of the neural correlates of general knowledge, we aimed to test two major hypotheses. First, assuming that the neural fundaments of general knowledge are the synapses between cortical neurons that are formed and modified during memory formation, we conjectured that general knowledge should be positively correlated with GMV. However, the simplified presumption that higher cortex volume may be equated with more storage capacity for general knowledge was not supported by our results. Initially, we observed a significant positive correlation between general knowledge and GMV. This structure-function relationship remained stable even after controlling for the effects of age and sex, two variables that have previously been shown to be strongly associated with general knowledge. However, after using Bonferroni correction to account for multiple comparisons, the association between general knowledge and GMV failed to reach statistical significance. Moreover, this was reflected in the results obtained by multiple regression analyses. GMV did not exhibit a unique contribution in the prediction of general knowledge. Most likely, this indicates a spurious correlation created by confounding effects of other neural predictors. It is conceivable that a great amount of explained variance in general knowledge is shared by GMV and $\mathrm{NET}_{\text {struc }}$ due to both variables being strongly correlated with each other (Table 2). Because GMV turned out to be a significant predictor of fluid intelligence, at least in the male subgroup, but not of general knowledge, one might conclude that the volume of the cortex, and hence the amount of neurons and neuropil, serves as a better predictor of problem solving abilities (Ritchie et al., 2015) than information storage abilities. Nevertheless, one has to consider the substantial sex differences that are tied to volumetric measures and their relation to cognitive performance.

Our second hypothesis revolved around the idea that the successful formation and retrieval of general knowledge is heavily dependent on the quality of a widely distributed brain network that allows for an efficient integration of information. It has been shown that object categories are represented by a continuous and distributed semantic map spanning the cortical surface (Huth, de Heer, Griffiths, Theunissen, \& Gallant, 2016; Huth, Nishimoto, Vu, \& Gallant, 2012). As a consequence, knowledge that combines diverse object categories is likely to be stored in the form of differently encoded information fragments distributed across the whole cortex. It is conceivable that the efficacy of storage and retrieval mechanisms operating within the scope of such a network can be quantified by measures of brain network connectivity like $\mathrm{NET}_{\text {struc }}$ or $\mathrm{NET}_{\text {func }}$. In fact, we observed a significant association between general knowledge and $\mathrm{NET}_{\text {struc }}$, indicating that brains comprising a very efficiently connected fibre network indeed hold more information. Contrary to the aforementioned analysis of GMV, this structurefunction relationship remained statistically significant even after controlling for multiple comparisons as well as the effects of age and sex. Moreover, the results of multiple regression analysis, with $\mathrm{NET}_{\text {struc }}$ remaining as the only significant predictor, also ruled out the possibility that the association between structural brain network connectivity and general knowledge was due to confounding effects of other brain properties. The association even remained stable after expanding the regression model by including fluid intelligence as a potential predictor of general knowledge. As suggested by cognitive investment theory, fluid intelligence had a unique contribution in predicting general knowledge, thereby adding to the overall variance explained by the regression model. However, this did not alter the association between $\mathrm{NET}_{\text {struc }}$ and general knowledge in any substantial way. This outcome is crucial to the claim that our findings represent distinguishable neural fundaments of general knowledge and fluid intelligence. Intelligence has been linked to structural brain network connectivity in the past (Li et al., 2009), and our data show a positive correlation between fluid intelligence and general knowledge. Therefore, it would have been possible that the statistically significant association between general knowledge and $\mathrm{NET}_{\text {struc }}$ merely represented a spurious relationship arising from confounding correlations with fluid intelligence. However, our supplemental analysis was able to rule out this scenario.

Because of the correlative nature of the study at hand, one has to be careful to derive any causal relations from the 
aforementioned findings. Final conclusions about the underlying causality and its direction have to remain speculative. Nevertheless, we believe that there are at least two justifiable interpretations of the observations made. The first revolves around the idea that all general knowledge is stored as information fragments, which are distributed across the whole brain and embedded into a complex fibre network. In this case, it is conceivable that every new piece of information entering long-term memory will alter the brain's fibre network by adding new connections or by discarding old connections representing obsolete information. The fibre network's morphology can thus be regarded as the result of knowledge acquisition, and the degree of its structural connectivity might represent the amount of general knowledge stored within it. This line of interpretation is inevitably linked to the assumption that BOWIT test scores mainly reflect the Gc component found in stratum II of the CHC model (Figure 1), that is, the depth and breadth of knowledge and skills that are valued by one's culture (W. Schneider \& McGrew, 2012). However, it has to be considered that knowledge tests like the BOWIT inventory not only measure the amount of general knowledge held by an individual but also provide an indirect estimate of long-term storage and retrieval abilities. These would correspond to the Glr component from the CHC model (Figure 1), which can be defined as the ability to store, consolidate, and retrieve information over periods of time measured in minutes, hours, days, and years (W. Schneider \& McGrew, 2012). This second line of interpretation thus embraces the idea that an efficiently connected fibre network does not necessarily represent the outcome of knowledge acquisition but rather an advantageous infrastructure for long-term storage of newly acquired information. From this, it follows that the fibre network's morphology influences the acquisition of knowledge but does not change as a result of the storage process. Finally, one could imagine that the two lines of interpretation are synergetic: an efficiently connected fibre network might emerge from information acquisition and storage in the past. At the same time, the presence of such a network is likely to foster the embedding of newly obtained information into memory traces of already existing general knowledge.

When performing multiple regression analysis for the whole sample, we observed a significant association between sex and general knowledge. However, the results from multiple regression analyses computed for both sexes separately did not show substantial differences compared with the whole-sample analysis and also revealed age and $\mathrm{NET}_{\text {struc }}$ as the only significant predictors. It is a noteworthy finding that the association between general knowledge and $\mathrm{NET}_{\text {struc }}$ was sex independent, although our data revealed that male subjects showed higher $\mathrm{NET}_{\text {struc }}$ values than female subjects and obtained higher test scores in the BOWIT inventory. These sex differences observed in our data correspond with findings reported by previous research. First, pioneering work by Ackerman et al. (2001) demonstrated that men are likely to perform better on knowledge questionnaires than women across a wide range of different knowledge domains. Second, a previous study by Ingalhalikar et al. (2014) reported substantial sex differences in overall brain connectivity with men showing more pronounced intrahemispheric fibre connections and women showing more pronounced interhemispheric fibre connections (Hanggi, Fovenyi, Liem, Meyer, \& Jancke, 2014). Interestingly, the structural connectome that was obtained by means of DTI fibre tracking included about $50 \%$ more intrahemispheric than interhemispheric edges after spurious connections were removed. Therefore, it is reasonable to assume that the more pronounced intrahemispheric connectivity within male brains leads to higher $\mathrm{NET}_{\text {struc }}$ values observed in the connectomes of male subjects compared with female subjects. As revealed by multiple regression analysis, the association between $\mathrm{NET}_{\text {struc }}$ and general knowledge is applicable to men and women alike. Therefore, we believe that our results, at least in parts, offer a possible neuroanatomical explanation for the consistent observation that men tend to perform better than women in tests of general knowledge (Ackerman et al., 2001). However, it goes without saying that sex differences in general knowledge are not elicited by neuroanatomical properties alone. According to Ackerman et al. (2001), sex differences in other factors such as ability selfestimates, personality traits, interests, or test anxiety are also likely to cause higher general knowledge test scores in men compared with women. Future studies might draw on our results and include these additional variables in their study design.

In summary, our study is the first to demonstrate that interindividual differences in general knowledge, one of the major components constituting Gc or crystallized intelligence, are tied to a neuroanatomical basis, which is dissociable from that of fluid intelligence. On the one hand, our results show that test scores obtained by general knowledge inventories are reflected in the efficiency of structural brain networks that can be quantified using state-of-the-art DTI methods. As a consequence, our findings raise the question whether structural brain network efficiency should be regarded as a predictor of the amount of general knowledge held by an individual, an individual's information storage and retrieval capacities, or even both. On the other hand, we were successful in confirming previous results, namely, that abilities in information processing, as measured by matrix reasoning test scores, are likely to be indicated by cortex volume and functional network connectivity. Importantly, it has to be considered that our sample was mainly composed of German university students who are not representative of the European population in terms of age, educational background, or ethnic composition. Therefore, one has to be careful when drawing conclusions about the general population based on our results.

In conclusion, our results provide first evidence of neuroanatomical correlates linked to general knowledge and add a missing piece to the mosaic constituting the biological foundation of cognitive performance, which still happens to be largely incomplete. Given the complexity of the human mind, it comes as no surprise that theories trying to capture its structure, such as the CHC model, are constituted of numerous components reflecting various aspects of cognitive 
ability. Another layer of complexity is added when taking into account that each of these many cognitive abilities is influenced by a multitude of different brain characteristics all contributing small amounts to the overall outcome. Thus, the endeavour of completely disentangling the relationships between the myriad of features constituting our neural substrate and the vast array of cognitive abilities may seem like a task that borders on sheer impossibility at times. Nevertheless, we hope that our findings encourage more neuroscientific research focused on general knowledge and other mainly unexplored yet exciting aspect of the human mind.

\section{AUTHOR CONTRIBUTIONS}

E. G. and O. G. conceived the project and supervised the experiments. E. G., C. F., C. S., and O. G. designed the experiments. C. F., C. S., and P. F. performed the experiments. E. G., C. F., M. C. V., and R. H. analysed the data. E. G., C. F., and O. G. wrote the manuscript, and all authors edited the manuscript.

\section{ACKNOWLEDGEMENTS}

This work was supported by the Deutsche Forschungsgemeinschaft (DFG) grant number Gu227/16 and the MERCUR Foundation grant number An-20150044. The authors thank Axel Kohler for his valuable comments on previous versions of this work as well as all research assistants for their support during behavioural measurements. Further, the authors thank Lara Schlaffke, Martijn Froeling, and PHILIPS Germany (Burkhard Mädler) for the scientific support with the MRI measurements as well as Tobias Otto for technical support.

\section{PREREGISTRATION STATEMENT}

The hypotheses included in this manuscript were not preregistered.

\section{SAMPLING STATEMENT}

The required sample size was not estimated a priori. Instead, we collected data from a reasonably large sample and computed the achieved power post hoc using G*Power (see the Methods section).

\section{OPEN MATERIAL STATEMENT}

The Methods section features a detailed description of all procedures applied and all measures assessed in this study. Parts of this dataset, including MRI data and/or behavioural data, have appeared in the following papers:

Schmitz, J., Fraenz, C., Schlüter, C., Friedrich, P., Jung, R. E., Güntürkün, O., ... Ocklenburg, S. (2019). Hemispheric asymmetries in cortical gray matter microstructure identified by neurite orientation dispersion and density imaging. Neurolmage, 189, 667-675.

Anderson, C., Gerding, W. M., Fraenz, C., Schlüter, C., Friedrich, P., Raane, M., ... Genç, E. (2018). PLP1 and CNTN1 gene variation modulates the microstructure of human white matter in the corpus callosum. Brain Structure and Function, 1-13.

Genç, E., Fraenz, C., Schlüter, C., Friedrich, P., Hossiep, R., Voelkle, M. C., ... Jung, R. E. (2018). Diffusion markers of dendritic density and arborization in gray matter predict differences in intelligence. Nature Communications, 9, 1905.

Ocklenburg, S., Friedrich, P., Fraenz, C., Schlüter, C., Beste, C., Güntürkün, O., \& Genç, E. (2018). Neurite architecture of the planum temporale predicts neurophysiological processing of auditory speech. Science Advances, 4, eaar6830.

Ocklenburg, S., Anderson, C., Gerding, W. M., Fraenz, C., Schlüter, C., Friedrich, P., ... Epplen, J. T. (2018). Myelin water fraction imaging reveals hemispheric asymmetries in human white matter that are associated with genetic variation in PLP1. Molecular Neurobiology, 1-14.

Schlüter, C., Fraenz, C., Pinnow, M., Friedrich, P., Güntürkün, O., $\&$ Genç, E. (2018). The structural and functional signature of action control. Psychological Science, 29, 1620-1630.

Friedrich, P., Ocklenburg, S., Heins, N., Schlüter, C., Fraenz, C., Beste, C., ... Genç, E. (2017). Callosal microstructure affects the timing of electrophysiological left-right differences. NeuroImage, 163, 310-318.

Schlüter, C., Fraenz, C., Pinnow, M., Voelkle, M. C., Güntürkün, O., \& Genç, E. (2018). Volition and academic achievement: Interindividual differences in action control mediate the effects of conscientiousness and sex on secondary school grading. Motivation Science, 4, 262.

\section{OPEN DATA STATEMENT}

Because the data included in this study are part of an ongoing research project, they are not made openly accessible yet. However, we plan on doing so once data collection and preprocessing are finished, presumably at the end of 2020 .

\section{REPRODUCIBLE SCRIPT STATEMENT}

Apart from the Brain Connectivity Toolbox (Rubinov \& Sporns, 2010) that is openly accessible via https://sites. google.com/site/bctnet/, the data analysis described in this manuscript does not require any special statistical code.

\section{EFFECTS STATEMENT}

In this manuscript, we report basic descriptive statistics, effect sizes, exact $p$-values, and 95\% confidence (credible) intervals. Further, we provide tables with descriptives of and correlations between all measures. Reliability estimates are given for both the general knowledge questionnaire (BOWIT) and the matrix reasoning intelligence test (BOMAT).

\section{SUPPORTING INFORMATION}

Additional supporting information may be found online in the Supporting Information section at the end of the article. 
Figure S1. Analysis of sex differences.

Figure S2. Results of additional multiple regression analyses separated by sex.

Figure S3. Additional regression model for the prediction of general knowledge.

\section{REFERENCES}

Ackerman, P. L. (1996). A theory of adult intellectual development: Process, personality, interests, and knowledge. Intelligence, 22, 227-257. https://doi.org/10.1016/S0160-2896(96)90016-1.

Ackerman, P. L. (2000). Domain-specific knowledge as the "dark matter" of adult intelligence: Gf/Gc, personality and interest correlates. Journals of Gerontology Series B-Psychological Sciences and Social Sciences, 55, P69-P84. https://doi.org/10.1093/ geronb/55.2.P69.

Ackerman, P. L., Bowen, K. R., Beier, M. E., \& Kanfer, R. (2001). Determinants of individual differences and gender differences in knowledge. Journal of Educational Psychology, 93, 797-825. https://doi.org/10.1037/0022-0663.93.4.797.

Basten, U., Hilger, K., \& Fiebach, C. J. (2015). Where smart brains are different: A quantitative meta-analysis of functional and structural brain imaging studies on intelligence. Intelligence, 51, 10-27. https://doi.org/10.1016/j.intell.2015.04.009.

Behrens, T. E. J., Berg, H. J., Jbabdi, S., Rushworth, M. F. S., \& Woolrich, M. W. (2007). Probabilistic diffusion tractography with multiple fibre orientations: What can we gain? NeuroImage, 34, 144-155. https://doi.org/10.1016/j.neuroimage.2006.09.018.

Behrens, T. E., Johansen-Berg, H., Woolrich, M. W., Smith, S. M., Wheeler-Kingshott, C. A., Boulby, P. A., Barker, G. J., ... Matthews, P. M. (2003). Non-invasive mapping of connections between human thalamus and cortex using diffusion imaging. $\mathrm{Na}$ ture Neuroscience, 6, 750-757. https://doi.org/10.1038/nn1075.

Behrens, T. E., Woolrich, M. W., Jenkinson, M., Johansen-Berg, H., Nunes, R. G., Clare, S., Matthews, P. M., ... Smith, S. M. (2003). Characterization and propagation of uncertainty in diffusion-weighted MR imaging. Magnetic Resonance in Medicine, 50, 1077-1088. https://doi.org/10.1002/mrm.10609.

Bratko, D., Butkovic, A., \& Chamorro-Premuzic, T. (2010). The genetics of general knowledge: A twin study from Croatia. Personality and Individual Differences, 48, 403-407. https://doi.org/ 10.1016/j.paid.2009.11.006.

Cattell, R. B. (1963). Theory of fluid and crystallized intelligence: A critical experiment. Journal of Educational Psychology, 54, 1-22. https://doi.org/10.1037/h0046743.

Choi, Y. Y., Shamosh, N. A., Cho, S. H., DeYoung, C. G., Lee, M. J., Lee, J. M., Kim, S. I., ... Lee, K. H. (2008). Multiple bases of human intelligence revealed by cortical thickness and neural activation. The Journal of Neuroscience, 28, 10323-10329. https:// doi.org/10.1523/JNEUROSCI.3259-08.2008.

Cohen, J. (1992). A power primer. Psychological Bulletin, 112, 155-159. https://doi.org/10.1037/0033-2909.112.1.155.

Cohen, M. X., Schoene-Bake, J. C., Elger, C. E., \& Weber, B. (2009). Connectivity-based segregation of the human striatum predicts personality characteristics. Nature Neuroscience, 12, 32-34. https://doi.org/10.1038/nn.2228.

Colom, R., Haier, R. J., Head, K., Alvarez-Linera, J.,Quiroga, M. A., Shih, P. C., \& Jung, R. E. (2009). Gray matter correlates of fluid, crystallized, and spatial intelligence: Testing the P-FIT model. Intelligence, 37(2), 124-135. https://doi.org/10.1016/j. intell.2008.07.007

Dale, A. M., Fischl, B., \& Sereno, M. I. (1999). Cortical surface-based analysis. I. Segmentation and surface reconstruction. NeuroImage, 9, 179-194. https://doi.org/10.1006/nimg. 1998.0395.
Deary, I. J., Penke, L., \& Johnson, W. (2010). The neuroscience of human intelligence differences. Nature Reviews. Neuroscience, 11, 201-211. https://doi.org/10.1038/nrn2793.

Desikan, R. S., Segonne, F., Fischl, B., Quinn, B. T., Dickerson, B. C., Blacker, D., Buckner, R. L., ... Killiany, R. J. (2006). An automated labeling system for subdividing the human cerebral cortex on MRI scans into gyral based regions of interest. NeuroImage, 31, 968-980. https://doi.org/10.1016/j.neuroimage.2006.01.021.

Dijkstra, E. W. (1959). A note on two problems in connexion with graphs. Numerische Mathematik, 1, 269-271. https://doi.org/ 10.1007/BF01386390.

Escorial, S., Román, F. J., Martínez, K., Burgaleta, M., Karama, S., \& Colom, R. (2015). Sex differences in neocortical structure and cognitive performance: A surface-based morphometry study. NeuroImage, $\quad 104, \quad 355-365 . \quad$ https://doi.org/10.1016/j. neuroimage.2014.09.035.

Faul, F., Erdfelder, E., Buchner, A., \& Lang, A. G. (2009). Statistical power analyses using $G^{*}$ power 3.1: Tests for correlation and regression analyses. Behavior Research Methods, 41, 1149-1160. https://doi.org/10.3758/BRM.41.4.1149.

Fischer, F. U., Wolf, D., Scheurich, A., \& Fellgiebel, A. (2014). Association of structural global brain network properties with intelligence in normal aging. PLOS ONE, 9.

Fischl, B., Sereno, M. I., \& Dale, A. M. (1999). Cortical surfacebased analysis. II: Inflation, flattening, and a surface-based coordinate system. NeuroImage, 9, 195-207. https://doi.org/10.1006/ nimg.1998.0396.

Fischl, B., van der Kouwe, A., Destrieux, C., Halgren, E., Segonne, F., Salat, D. H., Busa, E., ... Dale, A. M. (2004). Automatically parcellating the human cerebral cortex. Cerebral Cortex, 14, 11-22. https://doi.org/10.1093/cercor/bhg087.

Flanagan, D. P. (2013). The Cattell-Horn-Carroll theory of cognitive abilities. Encyclopedia of Special Education. https://doi. org/10.1002/9781118660584.ese0431.

Genç, E., Bergmann, J., Singer, W., \& Kohler, A. (2011). Interhemispheric connections shape subjective experience of bistable motion. Current Biology, 21, 1494-1499. https://doi.org/ 10.1016/j.cub.2011.08.003.

Genç, E., Bergmann, J., Tong, F., Blake, R., Singer, W., \& Kohler, A. (2011). Callosal connections of primary visual cortex predict the spatial spreading of binocular rivalry across the visual hemifields. Frontiers in Human Neuroscience, 5, 161.

Genc, E., Fraenz, C., Schluter, C., Friedrich, P., Hossiep, R., Voelkle, M. C., et al. (2018). Diffusion markers of dendritic density and arborization in gray matter predict differences in intelligence. Nature Communications, 9, 1905. https://doi.org/ 10.1038/s41467-018-04268-8.

Genc, E., Scholvinck, M. L., Bergmann, J., Singer, W., \& Kohler, A. (2015). Functional connectivity patterns of visual cortex reflect its anatomical organization. Cerebral Cortex.

Hanggi, J., Fovenyi, L., Liem, F., Meyer, M., \& Jancke, L. (2014). The hypothesis of neuronal interconnectivity as a function of brain size-A general organization principle of the human connectome. Frontiers in Human Neuroscience, 8, 915.

Hilger, K., Ekman, M., Fiebach, C. J., \& Basten, U. (2017). Efficient hubs in the intelligent brain: Nodal efficiency of hub regions in the salience network is associated with general intelligence. Intelligence, 60, 10-25. https://doi.org/10.1016/j.intell.2016.11.001.

Horn, J. L., \& Cattell, R. B. (1967). Age differences in fluid and crystallized intelligence. Acta Psychologica, 26, 107-129. https://doi.org/10.1016/0001-6918(67)90011-X.

Hossiep, R., Hasella, M., \& Turck, D. (2001). BOMAT—advanced -short version: Bochumer Matrizentest: Hogrefe, Verlag für Psychologie. Gottingen: Hogrefe.

Hossiep, R., \& Schulte, M. (2008). BOWIT: Bochumer Wissenstest. Gottingen: Hogrefe.

Huth, A. G., de Heer, W. A., Griffiths, T. L., Theunissen, F. E., \& Gallant, J. L. (2016). Natural speech reveals the semantic maps that tile human cerebral cortex. Nature, 532, 453-458. https:// doi.org/10.1038/nature17637. 
Huth, A. G., Nishimoto, S., Vu, A. T., \& Gallant, J. L. (2012). A continuous semantic space describes the representation of thousands of object and action categories across the human brain. Neuron, 76, 1210-1224. https://doi.org/10.1016/j. neuron.2012.10.014.

Ingalhalikar, M., Smith, A., Parker, D., Satterthwaite, T. D., Elliott, M. A., Ruparel, K., ... Verma, R. (2014). Sex differences in the structural connectome of the human brain. Proceedings of the National Academy of Sciences of the United States of America, 111(2), 823-828. https://doi.org/10.1073/pnas.1316909110

Ivkovic, M., Kuceyeski, A., \& Raj, A. (2012). Statistics of weighted brain networks reveal hierarchical organization and Gaussian degree distribution. PLoS ONE, 7, e35029. https://doi.org/10.1371/ journal.pone.0035029.

Jaeggi, S. M., Buschkuehl, M., Jonides, J., \& Perrig, W. J. (2008). Improving fluid intelligence with training on working memory. Proceedings of the National Academy of Sciences of the United States of America, 105, 6829-6833. https://doi.org/10.1073/ pnas.0801268105.

Jung, R. E., Brooks, W. M., Yeo, R. A., Chiulli, S. J., Weers, D. C., \& Sibbitt, W. L. Jr. (1999). Biochemical markers of intelligence: A proton MR spectroscopy study of normal human brain. Proceedings of the Biological Sciences, 266, 1375-1379. https://doi.org/10.1098/rspb.1999.0790.

Jung, R. E., \& Haier, R. J. (2007). The parieto-frontal integration theory (P-FIT) of intelligence: Converging neuroimaging evidence. Behavioral and Brain Sciences, 30, 135-154. https://doi. org/10.1017/S0140525X07001185.

Jung, R. E., Haier, R. J., Yeo, R. A., Rowland, L. M., Petropoulos, H., Levine, A. S., Sibbitt, W. L., et al. (2005). Sex differences in $\mathrm{N}$-acetylaspartate correlates of general intelligence: An ${ }^{1} \mathrm{H}-\mathrm{MRS}$ study of normal human brain. NeuroImage, 26, 965-972. https://doi.org/10.1016/j.neuroimage.2005.02.039.

Kanai, R., \& Rees, G. (2011). The structural basis of interindividual differences in human behaviour and cognition. Nature Reviews Neuroscience, 12, 231-242. https://doi.org/10.1038/ nrn3000.

Karama, S., Colom, R., Johnson, W., Deary, I. J., Haier, R., Waber, D. P., ... Grp, B. D. C. (2011). Cortical thickness correlates of specific cognitive performance accounted for by the general factor of intelligence in healthy children aged 6 to 18 . Neuroimage, 55(4), 1443-1453. https://doi.org/10.1016/J.Neuroimage.2011.01.016

Karbowski, J. (2007). Global and regional brain metabolic scaling and its functional consequences. BMC Biology, 5, 18. https:// doi.org/10.1186/1741-7007-5-18.

Kenkel, D. S. (1991). Health behavior, health knowledge, and schooling. Journal of Political Economy, 99, 287-305. https:// doi.org/10.1086/261751.

Kievit, R. A., van Rooijen, H., Wicherts, J. M., Waldorp, L. J., Kan, K. J., Scholte, H. S., \& Borsboom, D. (2012). Intelligence and the brain: A model-based approach. Cognitive Neuroscience, 3, 89-97. https://doi.org/10.1080/17588928.2011.628383.

Klein, D., Rotarska-Jagiela, A., Genc, E., Sritharan, S., Mohr, H., Roux, F., et al. (2014). Adolescent brain maturation and cortical folding: Evidence for reductions in gyrification. PLOS ONE, 9, e84914. https://doi.org/10.1371/journal.pone.0084914.

Klingberg, T. (2010). Training and plasticity of working memory. Trends in Cognitive Sciences, 14, 317-324. https://doi.org/ 10.1016/j.tics.2010.05.002.

Kvist, A. V., \& Gustafsson, J. E. (2008). The relation between fluid intelligence and the general factor as a function of cultural background: A test of Cattell's investment theory. Intelligence, 36, 422-436. https://doi.org/10.1016/j.intell.2007.08.004.

Latora, V., \& Marchiori, M. (2001). Efficient behavior of smallworld networks. Physical Review Letters, 87. https://doi.org/ 10.1103/PhysRevLett.87.198701.

Leuba, G., \& Kraftsik, R. (1994). Changes in volume, surface estimate, three-dimensional shape and total number of neurons of the human primary visual cortex from midgestation until old age. Anatomy and Embryology, 190, 351-366.
Li, Y., Liu, Y., Li, J., Qin, W., Li, K., Yu, C., et al. (2009). Brain anatomical network and intelligence. PLoS Computational Biology, 5, e1000395. https://doi.org/10.1371/journal.pcbi.1000395.

Liepmann, D., Beauducel, A., Brocke, B., \& Amthauer, R. (2007). Intelligenz-Struktur-Test $2000 R$ (IST 2000 R). Manual (2. erweiterte und überarbeitete Aufl.). Göttingen: Hogrefe.

Lynn, R., Irwing, P., \& Cammock, T. (2002). Sex differences in general knowledge. Intelligence, 30, 27-39.

Lynn, R., Ivanec, D., \& Zarevski, P. (2009). Sex differences in general knowledge domains. Collegium Antropologicum, 33, 515-520.

Lynn, R., Wilberg, S., \& Margraf-Stiksrud, J. (2004). Sex differences in general knowledge in German high school students. Personality and Individual Differences, 37, 1643-1650. https://doi. org/10.1016/j.paid.2004.02.018.

Martinez, K., Janssen, J., Pineda-Pardo, J. A., Carmona, S., Roman, F. J., Aleman-Gomez, Y., et al. (2017). Individual differences in the dominance of interhemispheric connections predict cognitive ability beyond sex and brain size. NeuroImage, 155, 234-244. https://doi.org/10.1016/j.neuroimage.2017.04.029.

McDaniel, M. A. (2005). Big-brained people are smarter: A metaanalysis of the relationship between in vivo brain volume and intelligence. Intelligence, 33, 337-346. https://doi.org/10.1016/j. intell.2004.11.005.

McGrew, K. S. (2005). The Cattell-Horn-Carroll theory of cognitive abilities: Past, present, and future.

Narr, K. L., Woods, R. P., Thompson, P. M., Szeszko, P., Robinson, D., Dimtcheva, T., et al. (2007). Relationships between IQ and regional cortical gray matter thickness in healthy adults. Cerebral Cortex, 17, 2163-2171. https://doi.org/10.1093/cercor/bhl125.

Oelhafen, S., Nikolaidis, A., Padovani, T., Blaser, D., Koenig, T., \& Perrig, W. J. (2013). Increased parietal activity after training of interference control. Neuropsychologia, 51, 2781-2790. https:// doi.org/10.1016/j.neuropsychologia.2013.08.012.

Oldfield, R. C. (1971). The assessment and analysis of handedness: The Edinburgh inventory. Neuropsychologia, 9, 97-113. https:// doi.org/10.1016/0028-3932(71)90067-4.

Oswald, W. D., \& Roth, E. (1987). Der Zahlen-Verbindungs-Test (ZVT). Göttingen; Toronto; Zürich: Hogrefe Verlag fuer Psychologie.

Pakkenberg, B., \& Gundersen, H. J. G. (1997). Neocortical neuron number in humans: Effect of sex and age. Journal of Comparative Neurology, 384, 312-320. https://doi.org/10.1002/ (SICI)1096-9861(19970728)384:2<312::AID-CNE10>3.0. $\mathrm{CO} ; 2-\mathrm{K}$.

Penke, L., Maniega, S. M., Bastin, M. E., Hernandez, M. C. V., Murray, C., Royle, N. A., ... Deary, I. J. (2012). Brain white matter tract integrity as a neural foundation for general intelligence. Molecular psychiatry, 17(10), 1026-1030. https:// doi.org/10.1038/Mp.2012.66

Pfleiderer, B., Ohrmann, P., Suslow, T., Wolgast, M., Gerlach, A. L., Heindel, W., \& Michael, N. (2004). N-Acetylaspartate levels of left frontal cortex are associated with verbal intelligence in women but not in men: A proton magnetic resonance spectroscopy study. Neuroscience, 123, 1053-1058. https://doi.org/ 10.1016/j.neuroscience.2003.11.008.

Pietschnig, J., Penke, L., Wicherts, J. M., Zeiler, M., \& Voracek, M. (2015). Meta-analysis of associations between human brain volume and intelligence differences: How strong are they and what do they mean? Neuroscience and Biobehavioral Reviews, 57, 411-432. https://doi.org/10.1016/j.neubiorev.2015.09.017.

Posthuma, D., De Geus, E. J. C., Baare, W. F. C., Pol, H. E. H., Kahn, R. S., \& Boomsma, D. I. (2002). The association between brain volume and intelligence is of genetic origin. Nature Neuroscience, 5, 83-84. https://doi.org/10.1038/nn0202-83.

Rae, C., Digney, A. L., McEwan, S. R., \& Bates, T. C. (2003). Oral creatine monohydrate supplementation improves brain performance: A double-blind, placebo-controlled, cross-over trial. Proceedings of the Royal Society B-Biological Sciences, 270, 2147-2150. https://doi.org/10.1098/rspb.2003.2492. 
Raven, J., Court, JH, \& Raven, J. (1990). Coloured progressive matrices. Manual for Raven's Progressive Matrices and Vocabulary Scales.

Raymond, M., \& Pontier, D. (2004). Is there geographical variation in human handedness? Laterality, 9, 35-51. https://doi.org/ $10.1080 / 13576500244000274$.

Regan, P. C., \& Joshi, A. (2003). Ideal partner preferences among adolescents. Social Behavior and Personality, 31, 13-20. https://doi.org/10.2224/sbp.2003.31.1.13.

Ritchie, S. J., Booth, T., Valdes Hernandez, M. D., Corley, J., Maniega, S. M., Gow, A. J., ... Deary, I. J. (2015). Beyond a bigger brain: Multivariable structural brain imaging and intelligence. Intelligence, 51, 47-56. https://doi.org/10.1016/j. intell.2015.05.001

Rolfhus, E. L., \& Ackerman, P. L. (1996). Self-report knowledge: At the crossroads of ability, interest, and personality. Journal of Educational Psychology, 88, 174-188. https://doi.org/10.1037/ 0022-0663.88.1.174.

Rolfhus, E. L., \& Ackerman, P. L. (1999). Assessing individual differences in knowledge: Knowledge, intelligence, and related traits. Journal of Educational Psychology, 91, 511-526. https:// doi.org/10.1037/0022-0663.91.3.511.

Roman, F. J., Abad, F. J., Escorial, S., Burgaleta, M., Martinez, K., Alvarez-Linera, J., et al. (2014). Reversed hierarchy in the brain for general and specific cognitive abilities: A morphometric analysis. Human Brain Mapping, 35, 3805-3818. https://doi.org/ 10.1002/hbm.22438.

Rubinov, M., \& Sporns, O. (2010). Complex network measures of brain connectivity: Uses and interpretations. NeuroImage, 52, 1059-1069. https://doi.org/10.1016/j.neuroimage.2009.10.003.

Ruigrok, A. N., Salimi-Khorshidi, G., Lai, M. C., Baron-Cohen, S., Lombardo, M. V., Tait, R. J., \& Suckling, J. (2014). A metaanalysis of sex differences in human brain structure. Neuroscience and Biobehavioral Reviews, 39, 34-50. https://doi.org/ 10.1016/j.neubiorev.2013.12.004.

Ryman, S. G., van den Heuvel, M. P., Yeo, R. A., Caprihan, A., Carrasco, J., Vakhtin, A. A., et al. (2014). Sex differences in the relationship between white matter connectivity and creativity. NeuroImage, 101, 380-389. https://doi.org/10.1016/j. neuroimage.2014.07.027.

Ryman, S. G., Yeo, R. A., Witkiewitz, K., Vakhtin, A. A., van den Heuvel, M., de Reus, M., ... Jung, R. E. (2016). Fronto-Parietal gray matter and white matter efficiency differentially predict intelligence in males and females. Hum Brain Mapp. https://doi. org/10.1002/hbm.23291

Salat, D. H., Greve, D. N., Pacheco, J. L., Quinn, B. T., Helmer, K. G., Buckner, R. L., \& Fischl, B. (2009). Regional white matter volume differences in nondemented aging and Alzheimer's disease. NeuroImage, 44, 1247-1258. https://doi.org/10.1016/j. neuroimage.2008.10.030.

Schneider, W., \& McGrew, K. (2012). The Cattell-Horn-Carroll model of intelligence. In D. Flanagan, \& P. Harrison (Eds.), Contemporary intellectual assessment: Theories, tests, and issues (3rd ed., pp. 99-144).

Schneider, W. J., \& Newman, D. A. (2015). Intelligence is multidimensional: Theoretical review and implications of specific cognitive abilities. Human Resource Management Review, 25, 12-27. https://doi.org/10.1016/j.hrmr.2014.09.004.

Takeuchi, H., Taki, Y., Nouchi, R., Hashizume, H., Sekiguchi, A., Kotozaki, Y., ... Kawashima, R. (2014). Effects of Multitasking-Training on Gray Matter Structure and Resting State Neural Mechanisms. Human Brain Mapping, 35(8), 3646-3660. https://doi.org/10.1002/hbm.22427

Tukey, J. W. (1977). Exploratory data analysis.

van den Heuvel, M. P., Stam, C. J., Kahn, R. S., \& Hulshoff Pol, H. E. (2009). Efficiency of functional brain networks and intellectual performance. The Journal of Neuroscience, 29, 7619-7624. https://doi.org/10.1523/JNEUROSCI.144309.2009.

Varley, R. (2007). Plasticity in high-order cognition: Evidence of dissociation in aphasia. Behavioral and Brain Sciences, 30, 171-172. https://doi.org/10.1017/S0140525X07001318.

Waltz, J. A., Knowlton, B. J., Holyoak, K. J., Boone, K. B., Mishkin, F. S., Santos, M. D., Thomas, C. R., et al. (1999). A system for relational reasoning in human prefrontal cortex. Psychological Science, 10, 119-125. https://doi.org/10.1111/14679280.00118 .

Wiltgen, B. J., Brown, R. A. M., Talton, L. E., \& Silva, A. J. (2004). New circuits for old memories: The role of the neocortex in consolidation. Neuron, 44, 101-108. https://doi.org/10.1016/j. neuron.2004.09.015.

Workman, L., \& Reader, W. (2014). Evolutionary psychology. Cambridge: Cambridge University Press. 